\title{
Wetting and drying of two-layer shallow-water flow in a moving vessel via semi-Lagrangian simulation
}

\author{
by M.R. Turner \& T.J. Bridges \\ Department of Mathematics, University of Surrey, \\ Guildford, Surrey GU2 7XH, UK
}

\begin{abstract}
- Abstract -
The sloshing motion of two inviscid, incompressible, immiscible, shallowwater fluid layers in a rectangular vessel with a rigid lid is considered. The vessel is forced to oscillate in a horizontal rectilinear motion, at the lowest resonant frequency of the system, such that the lower fluid hits the rigid lid causing a wetting-drying scenario. The two-layer shallow-water equations are solved using a conservative quasi-monotone semi-Lagrangian scheme which conserves the mass of the system. Two examples are considered: a low-fill example where the lower fluid does not interact with the upper-rigid lid, in order to validate the code against an existing Lagrangian particle path scheme, and a high-fill example where there is wetting and drying of the upper rigid lid. In both examples, results of the semi-Lagrangian simulations are compared to existing experimental data and good agreement is obtained.
\end{abstract}

— February 22, 2019-

\section{Introduction}

The challenge addressed in this paper is how to model the flow of a two-layer fluid inside a vessel allowing for wetting and drying of the upper lid. A schematic of the configuration of interest is shown in Figure 1 below. The most obvious and comprehensive approach to this problem is to use a full 2D or 3D simulation of the Euler equations with an accurate surface tracking model such as the Volume of Fluid method. This approach has been tested by the OWEL team in the context of ocean wave energy harvesters [1], but was found to be too computationally intensive and unsatisfactory for parametric studies. In this paper we propose a simplified model for two-layer flow including wetting and drying by using a shallow water model, thereby eliminating one flow direction.

Wetting and drying in the context of the shallow-water equations has been used before (e.g. the review of Balzano [2]). However these methods are predominantly Eulerian, and a second feature of interest in this study, after computational speed, is tracking of individual parcels of fluid, suggesting a Lagrangian Particle Path (LPP) viewpoint. The authors have had some success with LPP simulation of two-layer shallow water hydrodynamics [3]. The LPP scheme has excellent energy conservation properties, but the weaknesses 


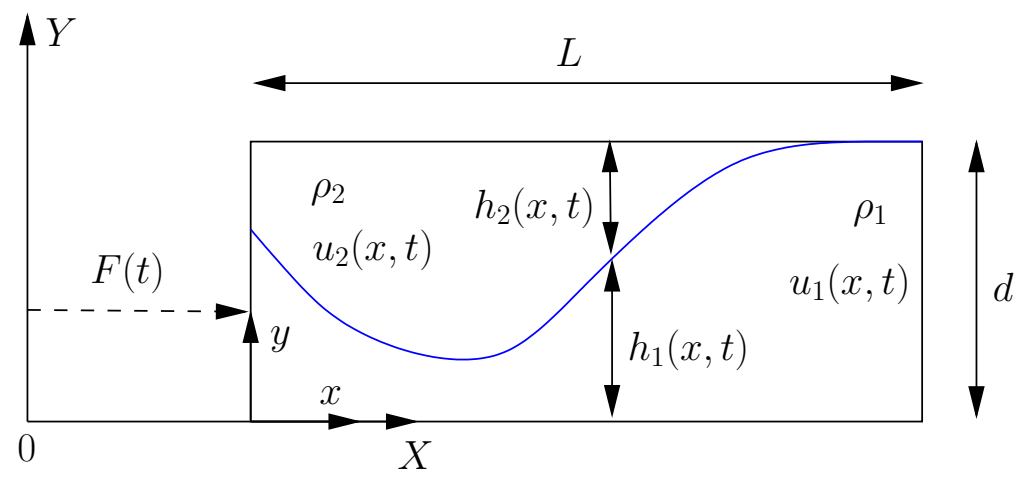

Figure 1: Schematic diagram of the two-layer system considered in this paper.

are complexity, due to layer matching, time-step restrictions, and the inability to simulate wetting/drying.

This impasse led the authors in the direction of semi-Lagrangian methods. The most successful semi-Lagrangian method is the Hamiltonian Particle Mesh (HPM) method of Frank and Reich [4]. Indeed there is a variant of HPM for two-layer shallow water hydrodynamics [5]. We tested this algorithm, but the two layers double the number of particles and computational times became prohibitive. Hence we were led in the direction of a class of semi-Lagrangian schemes without particles. That is, even though the advective terms are computed in a Lagrangian setting, only a mesh and not particles are used. This form of semi-Lagrangian scheme is widely used in numerical weather prediction and climate models [6, 7, 8].

This semi-Lagrangian method permits large time-steps, which can cause many Eulerian numerical schemes to be unstable, while retaining a fixed Eulerian grid throughout the simulation which aids the spatial discretization of the governing equations and enforcement of Eulerian constraints. Reviews of this scheme used here are given in [9] and [10]. A prototype of surface wetting by an advancing fluid is the dam break problem and it is known that semi-Lagrangian time-stepping is highly successful when applied to this problem [11].

There are drawbacks to the semi-Lagrangian scheme. Firstly, it is not mass conserving in its original form [10], and secondly, interpolation is required which can introduce spurious oscillations into the solution. A variety of methods have been proposed to make the semi-Lagrangian scheme conservative [12, 13, 14, 15, 16, 17]. In this paper we adopt the Conservative Quasi-Monotone semi-Lagrangian (CQMSL) scheme of [18]. This scheme is chosen as it is straightforward to implement, it does not itself introduce spurious oscillations, and it adds mass back into the system at points where the free-surface gradient is largest, which are the places where the error from applying interpolation schemes is largest.

Our motivation for studying wetting and drying of two-layer shallow-water flow is the deep ocean wave energy converter (WEC) patented by Offshore Wave Energy Ltd (OWEL). The OWEL WEC is essentially a rectangular box which has an opening at one end allowing waves to enter the device. The waves are then focused by the sloping walls of the device, which along with internal sloshing of the waves, causes the wave amplitudes to gradually grow and touch the rigid lid, pushing air through the power-take- 
off system, activating a turbine and generating electricity. The internal flow in the WEC is a two-layer flow of air over water, confined between an upper and lower surface, and experiments and CFD simulations of the OWEL device show the internal motion can be considered as predominantly two dimensional in most working environments [1]. As the inner dimensions of the OWEL WEC are $42 \mathrm{~m} \times 9 \mathrm{~m}$ (length $\times$ height) it is reasonable to assume the flow to be governed by two shallow-water layers of fluid of different densities. The purpose of this paper is to identify a numerical scheme which can simulate the wetting/drying of the upper rigid-lid, which can then be modified to more accurately simulate wave motion within the OWEL WEC. Hence we consider the simplified problem of a rectangular vessel with closed side walls, which is forced to horizontally translate periodically with a prescribed frequency and amplitude. Previous works investigating this simplified system (or similar systems), discussed below, were unable to adequately model the wetting and drying of the upper rigid lid, however the numerical scheme presented in this article does capture this feature of the flow. This wetting and drying is a critical feature required to model the power-take-off in the OWEL WEC.

The study of two-layer flows in open systems is a vast topic with a substantial literature, including works by [19, 20, 21, 22, 23, 24] to name a few. However of interest here are two-layer closed systems, where the literature is more limited. The most relevant numerical studies to the present work are those of [25] and [26] who studied similar systems to that in figure 1 using a numerical scheme based upon a class of high resolution wavepropagating finite volume methods, known as f-wave methods. These methods are quite versatile and can easily be extended to incorporate bottom topography [27] or multiple stratified layers [28]. Another relevant study by [3] developed a numerical scheme based upon the Lagrangian Particle Path (LPP) approach, in which a mapping, determined as part of the solution, coupled the label spaces in each layer, allowing the Eulerian rigid lid constraint

$$
h_{1}(x, t)+h_{2}(x, t)=d,
$$

to be satisfied at each streamwise position. Here $h_{1}$ and $h_{2}$ are the layer thicknesses in the lower and upper layers respectively, and $d$ is the fixed height of the vessel. However, neither the f-wave nor the LPP schemes could be modified to model a scenario where the lower layer touches the upper rigid lid, a crucial element to modelling the OWEL WEC.

Wetting and drying of one-layer shallow water hydrodynamics has been extensively studied, particularly for capturing the effect of flows up precluding topographies (e.g. [29, 30]). These studies are predominantly based on the Eulerian form of the governing equations. Using the full governing equations, there have been numerical studies which incorporate wetting/drying of the upper rigid lid, and these are usually based upon Computational Fluid Dynamics (CFD) models which solve the Navier-Stokes equations directly (incompressible and compressible), or the Reynolds averaged form of these equations. Such studies include [31, 32, 33, 34] and [35]. These CFD approaches are useful for understanding the characteristics of the flows, but the nature of the numerical scheme means they tend to be computationally expensive, hence resolutions tend to be limited in order to reduce run time. Also these schemes tend not to conserve the mass of the system, as seen in 35.

On the other hand, the CQMSL scheme applied to the two-layer shallow water equations allows for faster numerical evaluation whilst retaining good spatial resolution and mass conservation. Therefore faster parametric studies, targeted at optimizing features of the OWEL WEC such as its internal geometry, become feasible with this scheme. 
The current paper is laid out as follows. In 92 we derive the governing shallow-water equations for the time evolution of the free-surface, while in $\$ 3$ we discretize the equations and formulate the numerical scheme using the CQMSL approach. Results of this scheme are presented in \$4, in particular for a low-fill example with no wetting/drying in \$4.1 while a high-fill example including wetting/drying is found in \$4.2. Concluding remarks and discussion are given in $\$ 5$,

\section{Governing equations}

This article is concerned with two-layer, shallow-water sloshing in a closed vessel with rectangular cross-section, subject to a specified rectilinear forcing. A schematic diagram of the setup is depicted in figure 1. The vessel is a rigid body of length $L$ and height $d$ and is assumed to be filled will two immiscible, inviscid fluids of constant density $\rho_{1}$ and $\rho_{2}$ with $\rho_{1}>\rho_{2}$. Here, and in what follows, the subscripts 1 and 2 on the variables denote the lower and upper fluid layers respectively. The effect of the front and back faces of the vessel (found at $Z= \pm \frac{1}{2}$ ) are assumed to be negligible on the fluid motion, so that a quasi-two-dimensional analysis can be considered. As depicted in figure 1, we have an inertial frame of reference with Cartesian coordinates $\mathbf{X}=(X, Y)$ and a body frame system $\mathbf{x}=(x, y)$ which is fixed to the moving vessel. The two coordinate systems are linked via

$$
X=x+F(t), \quad Y=y,
$$

where $F(t)=\epsilon \sin (\omega t)$ or $\epsilon \cos (\omega t)$ is a stipulated, harmonic forcing with frequency $\omega$ and amplitude $\epsilon$.

The thickness of the fluid in each layer, denoted by $h_{i}(x, t)$ for $i=1,2$, is assumed to be small compared to length of the vessel, so that the flow in each layer, with corresponding horizontal velocity $u_{i}(x, t)$, can be modelled using the shallow-water equations. The Eulerian form of the mass conservation and momentum equations in each layer in the body frame is

$$
\begin{aligned}
\left(\rho_{1} h_{1}\right)_{t}+\left(\rho_{1} h_{1} u_{1}\right)_{x} & =0 \\
\left(\rho_{1} h_{1} u_{1}\right)_{t}+\left(\rho_{1} h_{1} u_{1}^{2}+\frac{1}{2} \rho_{1} g h_{1}^{2}\right)_{x} & =-\rho_{2} g h_{1} h_{2 x}-h_{1} p_{x}-\rho_{1} h_{1} \ddot{F} \\
\left(\rho_{2} h_{2}\right)_{t}+\left(\rho_{2} h_{2} u_{2}\right)_{x} & =0 \\
\left(\rho_{2} h_{2} u_{2}\right)_{t}+\left(\rho_{2} h_{2} u_{2}^{2}+\frac{1}{2} \rho_{2} g h_{2}^{2}\right)_{x} & =-\rho_{2} g h_{2} h_{1 x}-h_{2} p_{x}-\rho_{2} h_{2} \ddot{F},
\end{aligned}
$$

where $g>0$ is the gravitational constant, $p$ is the unknown pressure at the rigid lid $y=d$, the subscripts denote partial derivatives, and the over dots signify total derivatives with respect to $t$. Derivations of these equations can be found in [25] and [36]. Note that the mass of the fluid in each layer is denoted by $m_{f i}=\int_{0}^{L} \rho_{i} h_{i} \mathrm{~d} x$ for $i=1,2$.

As the two fluid system is confined between the impermeable surfaces at $y=0$ and $y=d$ the layer thicknesses must satisfy what we term the rigid lid constraint (1.1) at each $x$ position, while the boundary conditions at the side walls are the no penetration conditions

$$
u_{i}(0, t)=u_{i}(L, t)=0 \quad \text { for } \quad i=1,2
$$


We impose boundary conditions on $h_{i}$ at $x=0, L$, by satisfying the momentum equations, and as our fluids are inviscid we require the contact angle of the free-surface with the side walls to be $90^{\circ}$ when there is no wetting/drying, or $h_{1}=0$ or $h_{2}=0$ when the lower/upper surface is dry/wet. Therefore we impose the boundary conditions

$$
\left\{\begin{array}{l}
h_{1 x}=h_{2 x}=0, \\
h_{1}=d, h_{2}=0, \\
h_{1}=0, h_{2}=d,
\end{array} \quad \text { at } x=0, L .\right.
$$

Antuono et al. [37] noted, for the forced one-layer sloshing problem, that a consequence of requiring a $90^{\circ}$ contact angle at the side walls is that the forcing term $\ddot{F}$ in (2.4) and (2.6) needs to be modified such that

$$
\ddot{F}(t)=\left\{\begin{array}{ll}
\ddot{F} & \text { for } 0<x<L, \\
0 & \text { for } x=0, L
\end{array},\right.
$$

which we adopt in this work, along with the condition that $p_{x}=0$ at $x=0, L$. Note that [37] also show that this modulation of the forcing term conserves the momentum and energy in the final system and as such does not modify the original problem. A derivation of (2.8) along with the restriction (2.9) are derived in Appendix A, In this work we are specifically interested in the first two conditions of (2.8), i.e. we do not consider situations where $h_{1}=0$ and the bottom of the vessel becomes dry.

Another constraint on the system can be derived by adding (2.3) to (2.5) and noting that $h_{1 t}+h_{2 t}=0$ from (1.1), leading to $\left(h_{1} u_{1}+h_{2} u_{2}\right)_{x}=0$. This can be integrated with respect to $x$ and the boundary conditions (2.7) applied leading to the mass flux conservation condition

$$
U_{1}+U_{2}=0
$$

where we introduce the flux notation $U_{i}=h_{i} u_{i}$ for $i=1,2$. The introduction of the flux notation helps with simplifying future analysis.

The two-layer system (2.3)-(2.6) can be reduced to solving two equations only, by using the two constraints (1.1) and (2.10) to eliminate two of the variables, and by eliminating $p_{x}$. In this paper we choose to eliminate the lower layer variables $h_{1}=d-h_{2}$ and $u_{1}=h_{2} u_{2} / h_{1}$ and work solely with the upper layer variables. Therefore we need to solve the conservation of mass equation (2.5) along with the two momentum equations, which combine eliminating the pressure $\left(h_{2}(2.4)-h_{1}(2.6)\right)$ to give

$$
\begin{aligned}
U_{2 t}+u_{2} U_{2 x}+u_{2 x} U_{2} & -\frac{2 \rho_{1} d}{\left(d-h_{2}\right) \alpha} U_{2} U_{2 x}-\frac{\rho_{1} h_{2} h_{2 x}}{\left(d-h_{2}\right)^{2} \alpha} U_{2}^{2}+\frac{\rho_{1} h_{2 x}}{\alpha} u_{2} U_{2} \\
& +\frac{\left(\rho_{1}-\rho_{2}\right) h_{2}\left(d-h_{2}\right)}{\alpha}\left(g h_{2 x}-\ddot{F}\right)=0,
\end{aligned}
$$

where $\alpha=\rho_{1} h_{2}+\rho_{2}\left(d-h_{2}\right)$. Therefore we need an effective numerical scheme to solve (2.5) and (2.11) along with the two boundary conditions on $u_{2}$ and $h_{2}$ from (2.7) and (2.8). The goal of this article is to not only devise a numerical scheme which solves these equations accurately, but which can also produce solutions where the upper rigid lid periodically wets and drys, i.e. when $h_{2} \rightarrow 0$ and then becomes non-zero again. 


\section{The semi-Lagrangian method}

The approach taken in this article is to solve the system of equations from 82 using the semi-Lagrangian time-stepping scheme. This scheme combines a Lagrangian formulation to discretize the convective derivative, but discretizes the forces and constraints on a fixed Eulerian spatial grid.

By writing the convective derivative in the upper layer as

$$
\frac{\mathrm{d}}{\mathrm{d} t}=\frac{\partial}{\partial t}+u_{2} \frac{\partial}{\partial x}
$$

equations (2.5) and (2.11) become

$$
\begin{aligned}
\frac{\mathrm{d} h_{2}}{\mathrm{~d} t}+h_{2} u_{2 x} & =0, \\
\frac{\mathrm{d} U_{2}}{\mathrm{~d} t}+u_{2 x} U_{2} & -\frac{2 \rho_{1} d}{\left(d-h_{2}\right) \alpha} U_{2} U_{2 x}-\frac{\rho_{1} h_{2} h_{2 x}}{\left(d-h_{2}\right)^{2} \alpha} U_{2}^{2}+\frac{\rho_{1} h_{2 x}}{\alpha} u_{2} U_{2} \\
& +\frac{\left(\rho_{1}-\rho_{2}\right) h_{2}\left(d-h_{2}\right)}{\alpha}\left(g h_{2 x}-\ddot{F}\right)=0,
\end{aligned}
$$

with

$$
\left\{\begin{array}{l}
u_{2}=0 \\
h_{2 x}=0, \text { or } h_{2}=0, \text { or } h_{2}=d \quad \text { at } x=0, L .
\end{array}\right.
$$

\subsection{Spatial and temporal discretization}

To solve the above system of equations, we discretize space using the $M+1$ evenly-spaced grid points

$$
x_{j}=\Delta x(j-1), \quad \Delta x=\frac{L}{M}, \quad j=1, \ldots, M+1,
$$

and a time step $\Delta t$ such that $t_{n}=n \Delta t$. From this we can define the notation $u_{j}^{n}=$ $u_{2}\left(x_{j}, t_{n}\right)$ and similarly $h_{j}^{n}=h_{2}\left(x_{j}, t_{n}\right)$.

To construct the Lagrangian time-stepping part of the scheme, we assume a calculated solution at $t=t_{n}$ and step forward in time to $t=t_{n+1}$ via the characteristics

$$
\frac{\mathrm{d} x}{\mathrm{~d} t}=u_{2}(x, t)
$$

That is, we wish to find $u_{j}^{n+1}$ and $h_{j}^{n+1}$ for each grid point $j=1, \ldots, M+1$. The difficulty with this approach is that the $x$ values at $t_{n}$ for the characteristic which passes through $x_{j}$ at $t=t_{n+1}$ might not lie exactly at a grid point, as shown in figure 2. The $x$ value at time $t_{n}$ for the characteristic which passes through $x_{j}$ at $t_{n+1}$ is termed the departure point for $x_{j}$ and is denoted by $x_{d j}^{n}$. The departure point is found by solving (3.16) subject to $x\left(t_{n+1}\right)=x_{j}$. We discretize (3.16) using an approach based upon the implicit midpoint rule with the Adams-Bashforth method to make the resulting difference equation second order in $\Delta t$ such that

$$
\frac{x_{j}-x_{d j}^{n}}{\Delta t}=\frac{1}{2}\left[3 u_{2}\left(\frac{1}{2}\left(x_{j}+x_{d j}^{n}\right), t_{n}\right)-u_{2}\left(\frac{1}{2}\left(x_{j}+x_{d j}^{n}\right), t_{n}-\Delta t\right)\right] .
$$




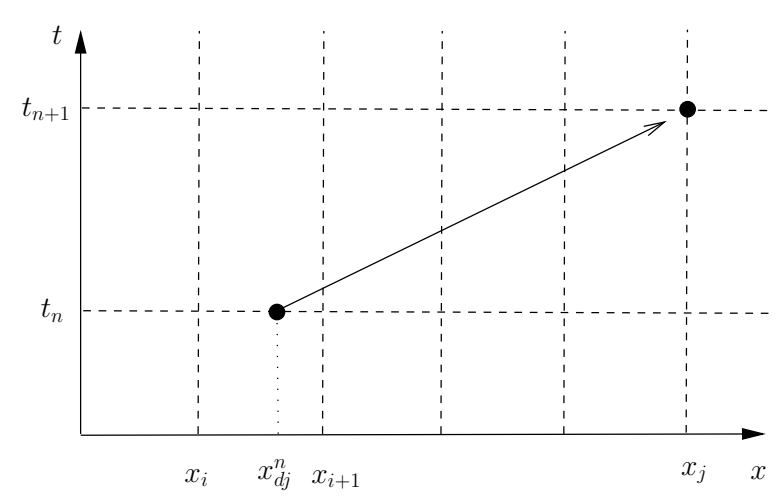

Figure 2: Semi-Lagrangian method of characteristics.

By writing $\beta_{j}=x_{j}-x_{d j}^{n}$ (3.17) can be written as the fixed point iterative formula

$$
\beta_{j}^{[r+1]}=\frac{\Delta t}{2}\left[3 u_{2}\left(x_{j}-\frac{1}{2} \beta_{j}^{[r]}, t_{n}\right)-u_{2}\left(x_{j}-\frac{1}{2} \beta_{j}^{[r]}, t_{n}-\Delta t\right)\right],
$$

for $\beta_{j}$, where $[r]$ denotes the iteration number. We iterate the above formula, for each $j=1, \ldots, M+1$, using linear interpolation to evaluate the RHS, until the absolute relative error between successive iterations is less than $10^{-10}$. For the first time step a first order backward Euler discretization is used.

Once the position of the departure points $x_{d j}^{n}$ for $j=1, \ldots, M+1$ are known, equations (3.12)-(3.14) can be discretized in time using the implicit mid-point rule giving the system

$$
\begin{aligned}
& h_{j}^{n+1}-\widetilde{h}_{j}^{n}+\frac{\Delta t}{4}\left(h_{j}^{n+1}+\widetilde{h}_{j}^{n}\right)\left(u_{x j}^{n+1}+\widetilde{u}_{x j}^{n}\right)=0, \\
& U_{j}^{n+1}-\widetilde{U}_{j}^{n}+\frac{\Delta t}{4}\left(u_{x j}^{n+1}+\widetilde{u}_{x j}^{n}\right)\left(U_{j}^{n+1}+\widetilde{U}_{j}^{n}\right)-\frac{\Delta t \rho_{1} d}{2\left(d-\frac{1}{2}\left(h_{j}^{n+1}+\widetilde{h}_{j}^{n}\right)\right) \alpha_{j}}\left(U_{j}^{n+1}+\widetilde{U}_{j}^{n}\right)\left(U_{x j}^{n+1}+\widetilde{U}_{x j}^{n}\right) \\
& -\frac{\Delta t \rho_{1}}{16\left(d-\frac{1}{2}\left(h_{j}^{n+1}+\widetilde{h}_{j}^{n}\right)\right)^{2} \alpha_{j}}\left(h_{j}^{n+1}+\widetilde{h}_{j}^{n}\right)\left(h_{x j}^{n+1}+\widetilde{h}_{x j}^{n}\right)\left(U_{j}^{n+1}+\widetilde{U}_{j}^{n}\right)^{2} \\
& +\frac{\rho_{1}}{8 \alpha_{j}}\left(h_{x j}^{n+1}+\widetilde{h}_{x j}^{n}\right)\left(u_{j}^{n+1}+\widetilde{u}_{j}^{n}\right)\left(U_{j}^{n+1}+\widetilde{U}_{j}^{n}\right) \\
& +\frac{\left(\rho_{1}-\rho_{2}\right)}{4 \alpha_{j}}\left(h_{j}^{n+1}+\widetilde{h}_{j}^{n}\right)\left(d-\frac{1}{2}\left(h_{j}^{n+1}+\widetilde{h}_{j}^{n}\right)\right)\left(g\left(h_{x j}^{n+1}+\widetilde{h}_{x j}^{n}\right)-2 \ddot{F}^{n+1 / 2}\right)=0
\end{aligned}
$$


for $j=2, \ldots, M$, where

$$
\begin{aligned}
\widetilde{h}_{j}^{n} & =h_{2}\left(x_{d j}^{n}, t_{n}\right), \\
\widetilde{u}_{j}^{n} & =u_{2}\left(x_{d j}^{n}, t_{n}\right), \\
U_{j}^{n+1} & =U_{2}\left(x_{j}, t_{n+1}\right), \\
\widetilde{U}_{j}^{n} & =U_{2}\left(x_{d j}^{n}, t_{n}\right), \\
\alpha_{j} & =\rho_{2} d+\frac{1}{2}\left(\rho_{1}-\rho_{2}\right)\left(h_{j}^{n+1}+\widetilde{h}_{j}^{n}\right), \\
\chi_{x j} & =\left.\frac{\partial \chi}{\partial x}\right|_{x_{j}} \text { for } \chi \in\left\{h^{n+1}, u^{n+1}, U^{n+1}\right\}, \\
\widetilde{\chi}_{x j} & =\left.\frac{\partial \chi}{\partial x}\right|_{x_{d j}^{n}} \text { for } \chi \in\left\{h^{n}, u^{n}, U^{n}\right\},
\end{aligned}
$$

i.e. the variables evaluated at the departure points are denoted by the tilded variables. The spatial derivatives have been discretized using second-order finite differences and the boundary conditions become

$$
\left\{\begin{array}{l}
u_{1}^{n+1}=u_{M+1}^{n+1}=0 \\
3 h_{1}^{n+1}-4 h_{2}^{n+1}+h_{3}^{n+1}=3 h_{M+1}^{n+1}-4 h_{M}^{n+1}+h_{M-1}^{n+1}=0 \\
\text { or } h_{k}^{n+1}=0 \text { or } h_{k}^{n+1}=d \text { for } k=1, M+1
\end{array}\right.
$$

using one-sided second-order spatial derivatives. Thus once the values of $\widetilde{h}_{j}^{n}, \widetilde{u}_{j}^{n}$ (and hence $\left.\widetilde{U}_{j}^{n}\right)$ and their derivatives are known, the $2 M+2$ nonlinear equations (3.18)-(3.20) can be solved via Newton iterations until the maximum absolute relative error is less than $10^{-10}$. In general the departure points do not fall at the Eulerian grid points, and hence the values of the tilded variables are estimated using interpolation. In this paper we use two interpolation methods for estimating these values, reasons for which will become apparent in 3.2 , a low resolution interpolate using linear interpolation, and a high resolution interpolate using Piecewise Cubic Hermite Interpolation [38]. This second interpolation scheme is exactly that implemented via the MATLAB ${ }^{\circledR}$ subroutine pchip.

In order to stop the computed result at $t=t_{n+1}$ becoming unphysical $\left(h_{j}^{n+1}<0\right.$ for any $j=1, \ldots, M+1$ ) we apply a threshold criteria where we set

$$
h_{j}^{n+1}=\left\{\begin{array}{ll}
h_{j}^{n+1} & \text { if } h_{j}^{n+1} \geq H^{*} \\
H^{*} & \text { if } h_{j}^{n+1}<H^{*}
\end{array},\right.
$$

and

$$
u_{j}^{n+1}=\left\{\begin{array}{ll}
u_{j}^{n+1} & \text { if } h_{j}^{n+1} \geq H^{*} \\
0 & \text { if } h_{j}^{n+1}<H^{*}
\end{array} .\right.
$$

Here $H^{*}>0$ is a numerical threshold value at which we assume the flow has become a single lower layer, hence its value identifies the thickness of the upper layer at which we assume this layer has vanished. Ideally we want this value to be close to zero, and in the results section we show that our model is actually insensitive to values of $H^{*} \in$ $\left[10^{-5}, 10^{-15}\right]$.

To aid with the stability of the numerical scheme, we adopt a variation on the weakly compressible form of the mass conservation equation used in the works of [39, 40, 37, 41]. 
We incorporate terms on the RHS of (2.3) and (2.5) of the form $\rho_{i} \delta h_{i x x}$ for $i=1,2$ where $\delta>0$ is a constant diffusive parameter. Consequently this leads to the additional terms

$$
-\frac{\rho_{2} \delta}{\alpha}\left(d-h_{2}\right) u_{2} h_{2 x x}+\frac{\rho_{1} \delta}{\alpha\left(d-h_{2}\right)} h_{2} U_{2} h_{2 x x}
$$

being added to the LHS of (3.13), which are then discretized using central differences. The parameter $\delta$ is included to smooth over the dispersive oscillations which occur on the hydraulic jumps during the solution procedure [39, 42].

The weakly compressible form of the mass conservation equations has been implemented and validated in the Smoothed Particle Hydrodynamics works of [39, 40, 37, 41]. In these papers $\delta$ represents a numerical diffusion with $\delta=c \Delta x \xi$ where $\Delta x$ is the grid step size in the $x$ direction, $c$ is the flow speed and $\xi$ is a non-dimensional constant. There, typical values of $\delta$ are of the order $10^{-2}$ to $10^{-1}$ for the problems considered. In this work our $\delta$ represents a continuum diffusion and we expect $\delta \propto h_{1}^{(0)}\left|u_{1 \max }\right|$ where $\left|u_{1 \max }\right|>0$ is the maximum flow velocity, therefore for each example considered in $\$ 4$ (fixed $h_{1}^{(0)}$ and $\epsilon$ etc.) $\delta$ is a constant. In this study we examine the dependence of our results on both $\delta$ and $H^{*}$ in order to identify their sensitivity. Ideally both these parameters should be as close to zero as possible, which turns out to be the case. When $\delta \neq 0$ the scheme is weakly non-mass conserving, but generally the semi-Lagrangian approach is not mass conserving, even when $\delta=0$. This can be resolved by considering the conservative quasi-monotone semi-Lagrangian formulation.

\subsection{The CQMSL scheme}

As discussed in the introduction, the semi-Lagrangian approach has two failings, firstly the interpolation scheme can introduce non-monotonic oscillations in the solution where none are expected to be present, and secondly the scheme does not conserve mass, again due to the interpolation scheme (see [10] for an excellent summary of the failings and resolution approaches for the semi-Lagrangian method). In this article we resolve both these issues by implementing the Conservative Quasi-Monotone Semi-Lagrangian (CQMSL) scheme of [43], in particular the mass conserving version of this algorithm presented in [18]. For full details of the algorithm the reader is directed to these two articles, and the references therein. Here we give a brief outline the algorithm for completeness.

The algorithm works on the basis that two solutions of (3.18)-(3.20) exist, a low resolution and a high resolution interpolate from 93.1 , which we term the low order and high order solution respectively. We denote these solutions with subscripts $L$ and $H$ respectively on the variables $h_{j}^{n+1}$ and $u_{j}^{n+1}$. The solution at the next time step is then a linear combination of these two solutions

$$
h_{j}^{n+1}=\gamma_{j} h_{H j}^{n+1}+\left(1-\gamma_{j}\right) h_{L j}^{n+1},
$$

where $0<\gamma_{j}, j=1, \ldots, M+1$ are constants to be determined. Note, the same combination also occurs for $u_{j}^{n+1}$ but we focus on $h_{j}^{n+1}$ as this is the quantity which needs to be adjusted in order to conserve mass. The CQMSL algorithm aims to make the coefficients $\gamma_{j}$ as close to one as possible (i.e. to form a solution as close to the high order solution as possible) without creating any new extrema and by conserving the mass of the system. 
The values for $\gamma_{j}$ are not usually written down explicitly, but are implicitly found by the following approach: evaluate the two functions

$$
\begin{aligned}
& H_{j}^{+}=\max \left\{h_{J}^{n}, h_{J+1}^{n}, h_{L j}^{n+1}: x_{J} \leq x_{d j}^{n} \leq x_{J+1}\right\}, \\
& H_{j}^{-}=\min \left\{h_{J}^{n}, h_{J+1}^{n}, h_{L j}^{n+1}: x_{J} \leq x_{d j}^{n} \leq x_{J+1}\right\},
\end{aligned}
$$

for $j=1, \ldots, M+1$, and then set

$$
\bar{H}_{j}= \begin{cases}H_{j}^{+} & \text {if } h_{H j}^{n+1}>H^{+} \\ H_{j}^{-} & \text {if } h_{H j}^{n+1}<H^{-} \\ h_{H j}^{n+1} & \text { otherwise. }\end{cases}
$$

Mass conservation then follows by defining the change in mass from the previous time-step to the new time-step as

$$
\Delta m_{f 2}=\frac{1}{2} \sum_{j=1}^{M}\left[\rho_{2}\left(\bar{H}_{j}+\bar{H}_{j+1}\right) \Delta x\right]-m_{f 2},
$$

where the trapezoidal rule has been used to evaluate the integral $m_{f 2}+\Delta m_{f 2}=\int_{0}^{L} \rho_{2} h_{2} \mathrm{~d} x$. If $\Delta m_{f 2}=0$ then mass is conserved and $h_{j}^{n+1}=\bar{H}_{j}$. Otherwise define the set of weighting functions $w_{j}$ and normalization factor $\lambda$ as

$$
w_{j}=\max \left\{0, \operatorname{sgn}\left(\Delta m_{f 2}\right)\left(h_{H j}^{n}-h_{L j}^{n}\right)^{3}\right\}, \quad \lambda=\frac{\Delta m_{f 2}}{\frac{1}{2} \sum_{j=1}^{M} \rho_{2}\left(w_{j}+w_{j+1}\right) \Delta x},
$$

where $\operatorname{sgn}(\cdot)$ is the sign function. If $w_{j}=0$ for all $j=1, \ldots, M+1$ then $h_{j}^{n+1}=\bar{H}_{j}$, else

$$
h_{j}^{n+1}=\bar{H}_{j}-\lambda w_{j}, \quad \text { for } \quad j=1, \ldots, M+1 .
$$

The calculation for $u_{j}^{n+1}$ at each time step is the same as above except with $\lambda \equiv 0$. Finally we again apply the threshold criteria (3.21) and (3.22) to check the physical nature of the solution. The results obtained using this algorithm, which conserve mass exactly, are presented in $\$ 4$.

\section{Results}

The results in this section focus on two examples of rectangular vessels forced to horizontally oscillate at the lowest resonant frequency of the system. Alemi Ardakani et al. [25] showed that the lowest natural frequency for free-sloshing in a two-layer flow in the shallow-water limit is

$$
\omega_{1}=\frac{n \pi}{L} \sqrt{\frac{g\left(\rho_{1}-\rho_{2}\right) h_{1}^{(0)} h_{2}^{(0)}}{\rho_{1} h_{2}^{(0)}+\rho_{2} h_{1}^{(0)}}}
$$

where $h_{i}^{(0)}$ is the mean fluid level for $i=1,2$. The first example in $\$ 4.1$ considers a case with no wetting/drying of the upper rigid lid, and hence provides a good test example for the semi-Lagrangian scheme, for which the results can be compared to the experiments of [44]. As there is no wetting/drying it also means that results can be directly compared to 
those of the two-layer Lagrangian Particle Path (LPP) code presented in [3]. The second example in $\$ 4.2$ considers a case where there is wetting/drying of the upper rigid lid, and results can be compared to the experimental results of [45]. Both sets of experimental results can also be found in the work of [35] who use a non-shallow water numerical scheme to compare with the experiments. In both sections the two fluids studied are water with $\rho_{1}=1025 \mathrm{kgm}^{3}$ and air with $\rho_{2}=1 \mathrm{kgm}^{3}$ giving a density ratio $\rho_{2} / \rho_{1} \approx 10^{-3}$.

\subsection{Low-fill example}

Here we consider a rectangular vessel of length $L=1.2 \mathrm{~m}$, height $d=0.6 \mathrm{~m}$, filled to a quiescent height $h_{1}^{(0)}=0.12 \mathrm{~m}\left(h_{2}^{(0)}=0.48 \mathrm{~m}\right)$ which has $F(t)=\epsilon \sin (\omega t)$, and is forced with frequency $\omega=2.839 \mathrm{~s}^{-1}$. With the smoothing parameter $\delta=0 \mathrm{~m}^{2} \mathrm{~s}^{-1}$, the small amplitude result with forcing amplitude $\epsilon=6 \times 10^{-4} \mathrm{~m}$ is given in figure 3. Despite the

(a)
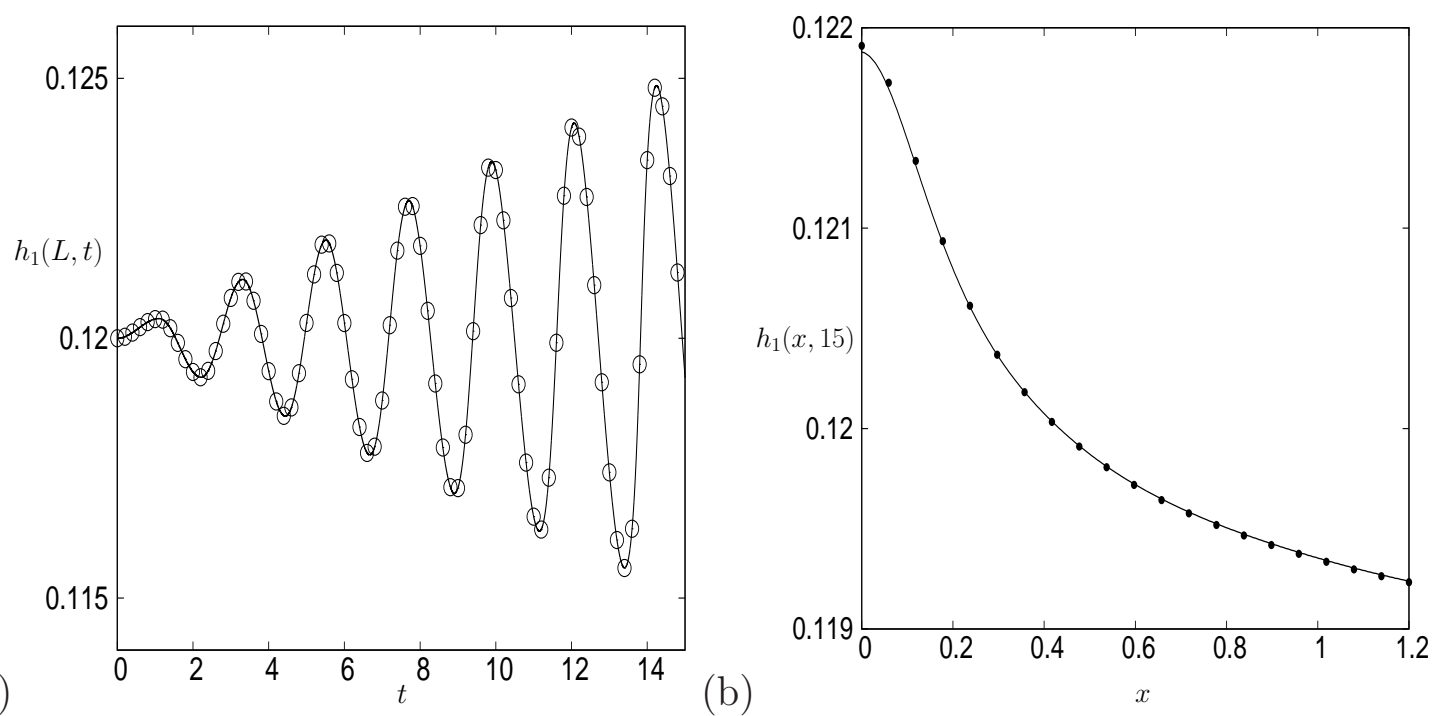

Figure 3: Plot of (a) $h_{1}(L, t)$ and (b) $h_{1}(x, 15)$ for the low-fill example when $L=1.2 \mathrm{~m}$, $d=0.6 \mathrm{~m}, h_{1}^{(0)}=0.12 \mathrm{~m}, \omega=2.839 \mathrm{~s}^{-1}, \epsilon=6 \times 10^{-4} \mathrm{~m}$ with simulation parameters $\delta=0 \mathrm{~m}^{2} \mathrm{~s}^{-1}, \delta t=10^{-3} \mathrm{~s}$ and $M=200$. The circles in panel (a) and the dots in panel (b) represent the results given by the LPP code of [3] with the same spatial and temporal discretization.

numerical scheme being formulated in terms of $h_{2}$, it is more intuitive to plot results in terms of $h_{1}=d-h_{2}$, as this indicates the position of the fluid interface as observed in experiments. These linear results show excellent agreement between the semi-Lagrangian code and the LPP code, which is designed to be energy conserving (using a symplectic numerical integrator). Figure 3(a) shows the amplitude of the fluid at the right-hand wall increasing, but not reaching a magnitude where nonlinear terms in the governing equations become significant.

In figure 4 the amplitude of the forcing is increased to $\epsilon=6 \times 10^{-3} \mathrm{~m}$. In this case, the nonlinear effects become significant and the internal sloshing wave steepens into a travelling hydraulic jump. Agreement between the semi-Lagrangian scheme and the LPP scheme is excellent up to $t \approx 9 \mathrm{~s}$, where the LPP scheme, and the semi-Lagrangian scheme 

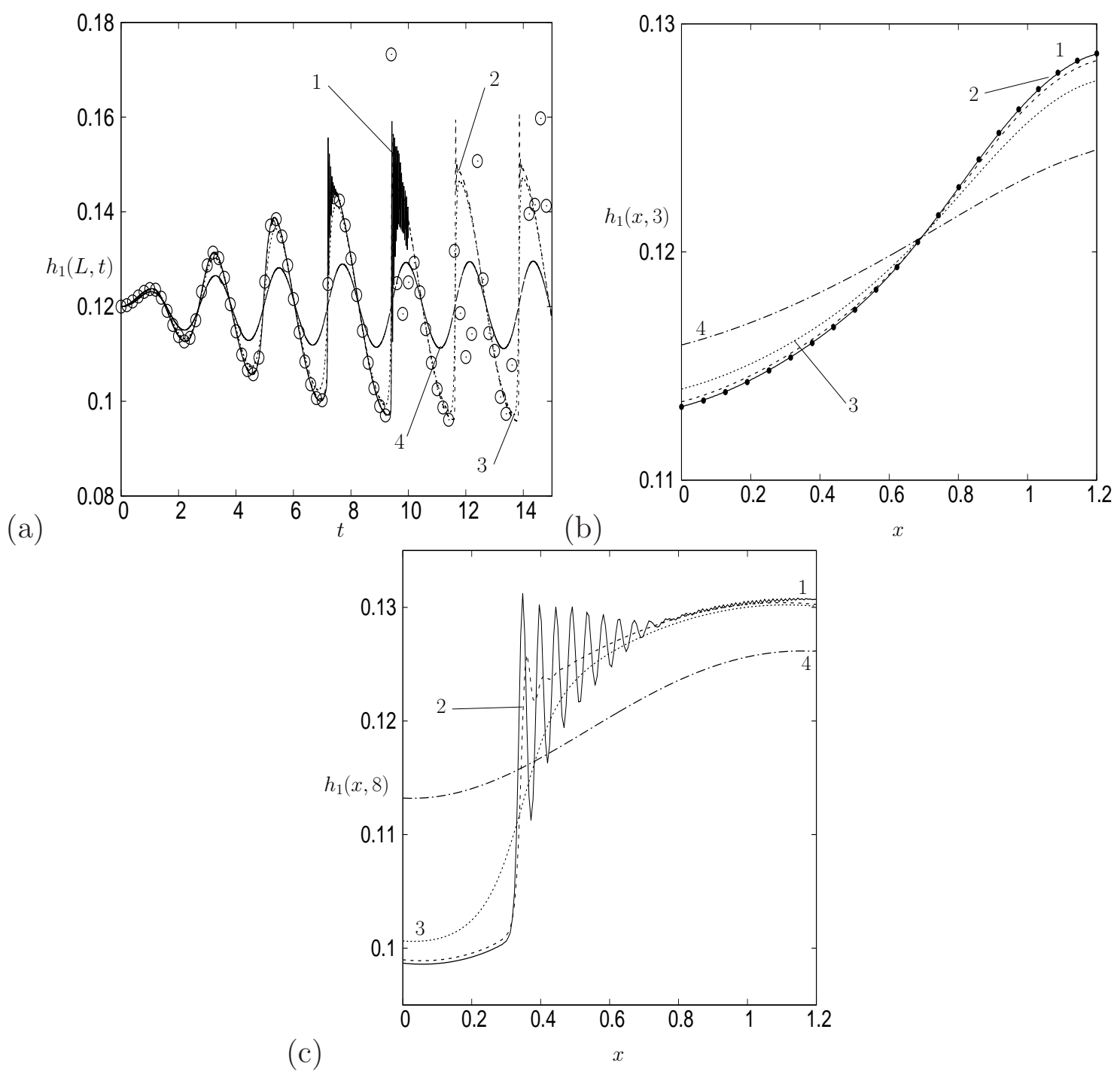

Figure 4: Plot of (a) $h_{1}(L, t)$, (b) $h_{1}(x, 3)$ and (b) $h_{1}(x, 8)$ for the low-fill example when $L=1.2 \mathrm{~m}, d=0.6 \mathrm{~m}, h_{1}^{(0)}=0.12 \mathrm{~m}, \omega=2.839 \mathrm{~s}^{-1}, \epsilon=6 \times 10^{-3} \mathrm{~m}$ with simulation parameters $\delta t=10^{-3} \mathrm{~s}, M=200$ and $\delta=0,10^{-3}, 10^{-2}, 10^{-1} \mathrm{~m}^{2} \mathrm{~s}^{-1}$ numbered $1-4$ respectively. The circles in panel (a) and the dots in panel (b) represent the results given by the LPP code of [3] with the same spatial and temporal discretization.

with $\delta=0 \mathrm{~m}^{2} \mathrm{~s}^{-1}$, begin to form fine scale oscillations, which in fact cause the semiLagrangian scheme to fail to converge around $t \approx 10 \mathrm{~s}$. These oscillations are numerical artifacts due to dispersive shock waves forming on the fluid interface as the steep wave front hits the side wall of the vessel and the free-surface 'flips through' [35]. At this point our shallow-water assumption breaks down due to the neglected vertical accelerations becoming significant, and this manifests itself in the solution via these oscillations. The dispersive shock waves can be clearly seen on result 1 of figure 4(c). The introduction of the smoothing term $\delta$ to the scheme smooths out these dispersive waves without significantly affecting the overall system behaviour for 'small enough' $\delta$. We see in figure 4 (a) and 4 (c) that $\delta=10^{-3} \mathrm{~m}^{2} \mathrm{~s}^{-1}$ is almost sufficient to completely remove the dispersive oscillations while leaving the side-wall elevation almost indistinguishable from the $\delta=0 \mathrm{~m}^{2} \mathrm{~s}^{-1}$ result. 
Increasing the value to $\delta=10^{-2} \mathrm{~m}^{2} \mathrm{~s}^{-1}$ has the small effect of reducing the side-wall elevation, but gives a fluid interface which is much smoother and more like a steep wave rather than a hydraulic jump. Clearly $\delta=10^{-1} \mathrm{~m}^{2} \mathrm{~s}^{-1}$ is too large to be considered as a suitable smoothing value and damps the waves too much. These results suggest a value of $\delta \in\left[10^{-3}, 10^{-2}\right] \mathrm{m}^{2} \mathrm{~s}^{-1}$ for this example, however this is explored further in the convergence results which follow.

\begin{tabular}{|l|r|l|l||l|r|l|l|}
\hline$M$ & $\Delta t$ & $I(\Delta t, M, L)$ & $h_{1}(0,10)$ & $M$ & $\Delta t$ & $I(\Delta t, M, L)$ & $h_{1}(0,10)$ \\
\hline 100 & $10^{-3}$ & 0.13236 & 0.06729 & 300 & $2 \times 10^{-4}$ & 0.14142 & 0.06569 \\
100 & $2 \times 10^{-4}$ & 0.13261 & 0.06911 & 300 & $10^{-4}$ & 0.13398 & 0.06589 \\
100 & $10^{-4}$ & 0.13205 & 0.07058 & 300 & $2 \times 10^{-5}$ & 0.13271 & 0.06589 \\
100 & $2 \times 10^{-5}$ & 0.13220 & 0.07075 & 300 & $10^{-5}$ & 0.13171 & 0.06589 \\
100 & $10^{-5}$ & 0.13239 & 0.07084 & 400 & $10^{-4}$ & 0.13086 & 0.06562 \\
200 & $10^{-3}$ & 0.14541 & 0.06590 & 400 & $2 \times 10^{-5}$ & 0.12983 & 0.06563 \\
200 & $2 \times 10^{-4}$ & 0.14988 & 0.06618 & 400 & $10^{-5}$ & 0.12895 & 0.06564 \\
200 & $10^{-4}$ & 0.14869 & 0.06698 & 500 & $10^{-4}$ & 0.12894 & 0.06550 \\
200 & $2 \times 10^{-5}$ & 0.14747 & 0.06706 & 500 & $2 \times 10^{-5}$ & 0.12797 & 0.06551 \\
200 & $10^{-5}$ & 0.14602 & 0.06707 & 500 & $10^{-5}$ & 0.12713 & 0.06552 \\
\hline
\end{tabular}

Table 1: Table of convergence data to accompany figure 5 ,

The convergence properties of the numerical scheme for the low-fill example with $\epsilon=6 \times 10^{-2} \mathrm{~m}$ are considered in figure 5(a), and table 1. Here we plot and give values of

$$
I(\Delta t, M, x)=\int_{0}^{15} h_{1}(x, t) \mathrm{d} t,
$$

at $x=L$ for various values of $\Delta t$ and $M$ and the table also gives values of $h_{1}(0,10)$. The results show that in this case the scheme converges in the limit of large $M$ and small $\Delta t$, but does so slowly. However, this slow convergence is due mainly to the nature of the integrand in (4.24), and the mass conservation part of the CQMSL scheme. When we plot $h_{1}(L, t)$ in figure 5 (b) for $\Delta t=5 \times 10^{-5} \mathrm{~s}$ and $M=100,200,300,400,500$ we see that all 5 results are very similar, with the $M=200,300,400$ and 500 results almost indistinguishable on this plot. If we consider the spatial form of the interfaces at $t=10 \mathrm{~s}$ in figure 5(c), we see the convergence more clearly, in fact for $M=300,400$ and 500 the interface profiles are almost identical. In figure 5(d) we plot $h_{1}(x, 10)$ for $M=400$ and $\Delta t=10^{-4}, 5 \times 10^{-5}$ and $10^{-5} \mathrm{~s}$ and we again note that all profiles are almost indistinguishable.

In figure 6 and table 2 we consider the convergence properties of the model for $\epsilon=$ $6 \times 10^{-2} \mathrm{~m}$ as a function of the diffusion parameter $\delta$. Table 2 along with the corresponding results in table 1 show that for each value of $\delta$ the model converges for large $M$ and small $\Delta t$, but does so more slowly for smaller $\delta$ values. The reason for this is again due to the flip through of the free-surface at the wall producing dispersive shock waves which are still present in the solution as seen in figure 6 for $\delta=10^{-3}$ and $2 \times 10^{-3}$. These results show that there exists a range of $\delta$ values for which the dispersive waves are removed from the solution, but where the shock solution is not over damped. This range of $\delta$ needs to be calculated via numerical experiment. For low-fill examples with no rigid lid interaction, the free-surface flip through at the wall means that we require $\delta=O\left(10^{-2}\right)$ to remove 


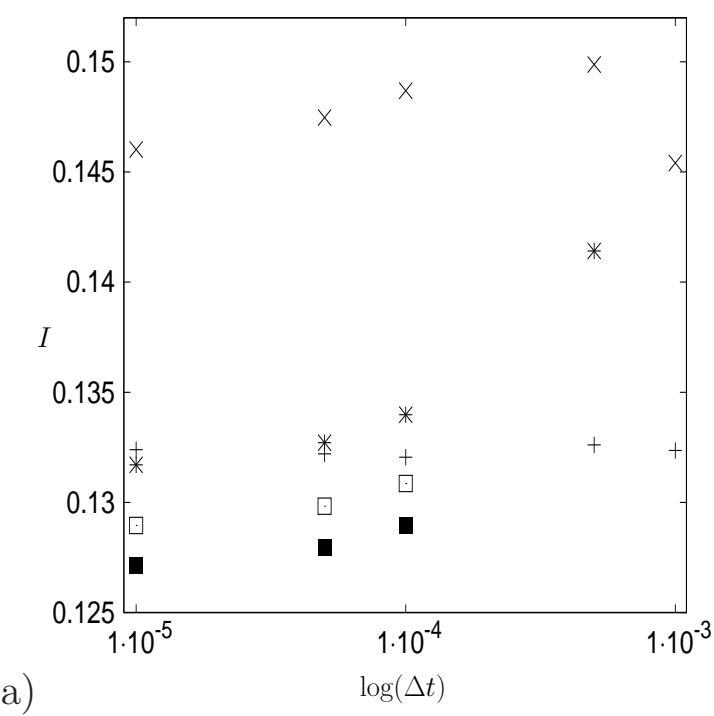

(a)

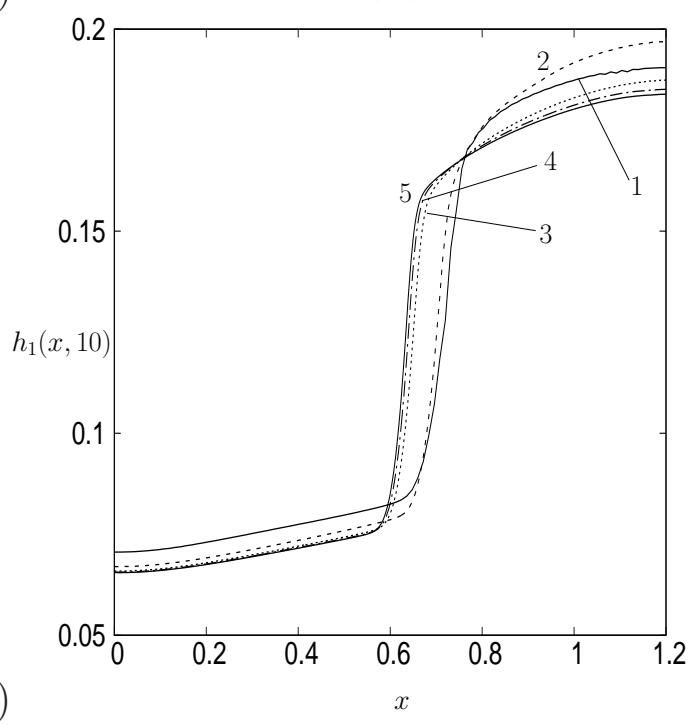

(b)
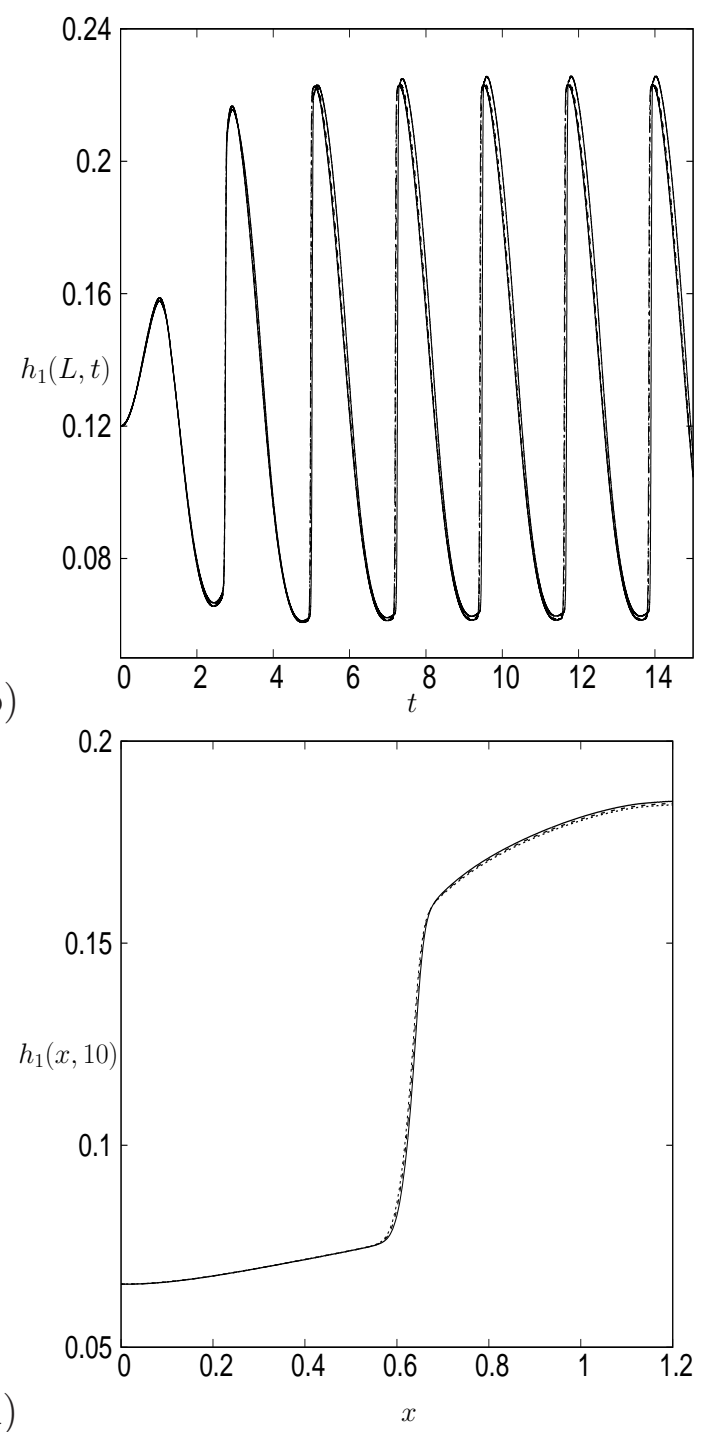

Figure 5: Plot of the convergence properties of the low-fill example with $L=1.2 \mathrm{~m}$, $d=0.6 \mathrm{~m}, h_{1}^{(0)}=0.12 \mathrm{~m}, \omega=2.839 \mathrm{~s}^{-1}, \epsilon=6 \times 10^{-2} \mathrm{~m}$ and $\delta=10^{-2} \mathrm{~m}^{2} \mathrm{~s}^{-1}$. Panel (a) plots $I(\Delta t, M, L)$ with (+) $M=100,(\times) M=200,(*) M=300$, ( $\square) M=400$ and (ם) $M=500$. Panel (b) plots $h_{1}(L, t)$ and panel (c) plots $h_{1}(x, 10)$ with $\Delta t=10^{-4} \mathrm{~s}$ and $M=100,200,300,400,500$ numbered 1-5 respectively. Panel (d) plots $h_{1}(x, 10)$ with $M=400$ and $\Delta t=10^{-4}, 5 \times 10^{-5}$ and $10^{-5} \mathrm{~s}$.

the dispersive shock waves, however, we will see in $\$ 4.2$ that when the effect of the rigid lid is significant, this flip through does not occur (see [35]) and so we find a smaller value of $\delta\left(=O\left(10^{-3}\right)\right)$ is sufficient, which means less numerical damping is required.

Finally, to compare our simulation results to experimental data, we need to convert fluid layer thickness data into pressure data. For this low-fill example we compare our results against the experimental results of [44], who present results at a pressure sensor attached to the bottom of their vessel close to the right-hand wall, $x=L$ (see [35] for a schematic diagram of the experimental setup). Thus we determine the pressure at this point via

$$
p_{L}(L, t)=\rho_{1} g h_{1}(L, t)+\rho_{2} g h_{2}(L, t),
$$




\begin{tabular}{|l|r||l|l|l||l|l|l|}
\hline$M$ & $\Delta t$ & $\begin{array}{l}I(\Delta t, M, L), \\
\delta=5 \times 10^{-3}\end{array}$ & $\begin{array}{l}I(\Delta t, M, L) \\
\delta=2 \times 10^{-3}\end{array}$ & $\begin{array}{l}I(\Delta t, M, L) \\
\delta=10^{-3}\end{array}$ & $\begin{array}{l}h_{1}(0,10) \\
\delta=5 \times 10^{-3}\end{array}$ & $\begin{array}{l}h_{1}(0,10) \\
\delta=2 \times 10^{-3}\end{array}$ & $\begin{array}{l}h_{1}(0,10) \\
\delta=10^{-3}\end{array}$ \\
\hline 300 & $5 \times 10^{-5}$ & 0.15795 & 0.13116 & 0.13120 & 0.06588 & 0.07020 & 0.07144 \\
300 & $2 \times 10^{-5}$ & 0.15801 & 0.13036 & 0.12788 & 0.06602 & 0.07007 & 0.07129 \\
300 & $10^{-5}$ & 0.15795 & 0.13066 & 0.12669 & 0.06605 & 0.07014 & 0.07128 \\
400 & $5 \times 10^{-5}$ & 0.15835 & 0.13659 & 0.13837 & 0.06554 & 0.06721 & 0.07000 \\
400 & $2 \times 10^{-5}$ & 0.15791 & 0.13366 & 0.13420 & 0.06562 & 0.06860 & 0.07012 \\
400 & $10^{-5}$ & 0.15768 & 0.13479 & 0.13295 & 0.06564 & 0.06824 & 0.07019 \\
500 & $5 \times 10^{-5}$ & 0.15414 & 0.14164 & 0.13939 & 0.06533 & 0.06551 & 0.06940 \\
500 & $2 \times 10^{-5}$ & 0.15299 & 0.14420 & 0.13760 & 0.06536 & 0.06651 & 0.06847 \\
500 & $10^{-5}$ & 0.15244 & 0.14717 & 0.13568 & 0.06538 & 0.06632 & 0.06857 \\
\hline
\end{tabular}

Table 2: Table of convergence data to accompany figure 6.
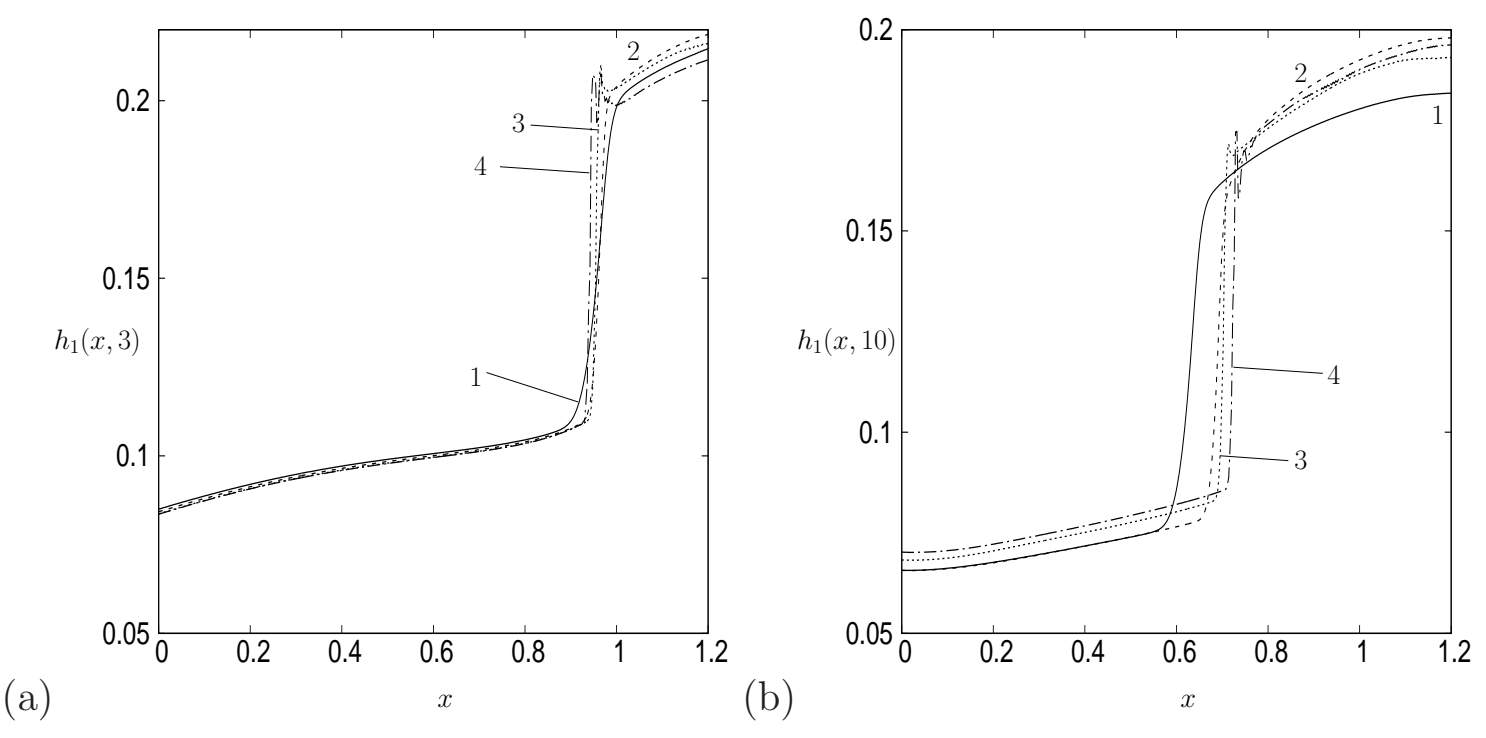

Figure 6: Plot of $h_{1}(x, t)$ for the low-fill example with $L=1.2 \mathrm{~m}, d=0.6 \mathrm{~m}, h_{1}^{(0)}=0.12 \mathrm{~m}$, $\omega=2.839 \mathrm{~s}^{-1}, \epsilon=6 \times 10^{-2} \mathrm{~m}, M=400$ and $\Delta t=10^{-5} \mathrm{~s}$ at (a) $t=3 \mathrm{~s}$ and (b) $t=10 \mathrm{~s}$. In each panel lines $1-4$ represent $\delta=\delta=10^{-2}, 5 \times 10^{-3}, 2 \times 10^{-3}$ and $10^{-3} \mathrm{~m}^{2} \mathrm{~s}^{-1}$ respectively.

which comes from the shallow-water assumption that the pressure is hydrostatic. In order to compare our shallow-water results to the fully two-dimensional experiments of [44] we set up our simulation such that the vessel has the same dimensions and with the same fluid depth. The experiment was conducted with a forcing frequency $\omega=3.611 \mathrm{~s}^{-1}$, but if we were to use this value in our shallow-water model we would not see a resonant response, and hence we would not encounter the resonant waves seen in the experiment. Therefore we force our simulation at the lowest shallow-water resonance for this problem of $\omega=2.839 \mathrm{~s}^{-1}$, from (4.23) in order to see a resonant response, and then we stretch the time points for the experimental data to equate the forcing frequency with that in our simulation. The comparison of $p_{L}(L, t)$ is given in figure 7 with the experimental data given by the dashed line. The results show good agreement in the magnitude of the pressure peaks and troughs. As expected the experimental data is noisier than the 


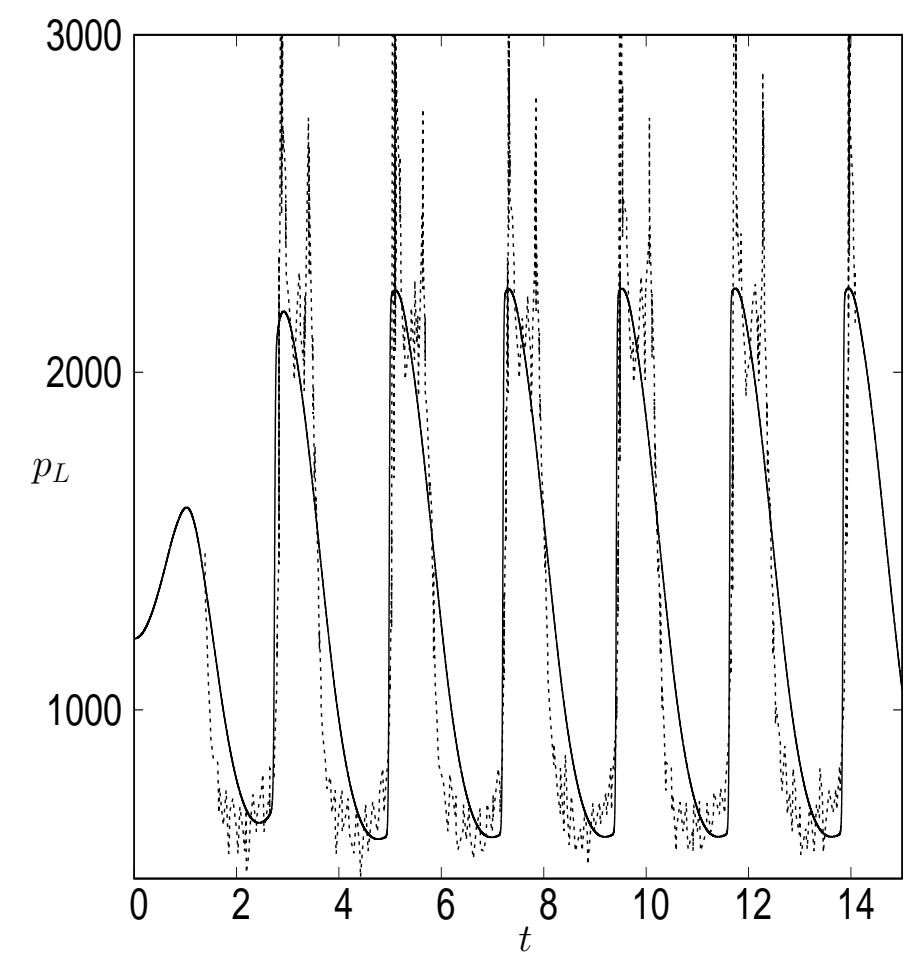

Figure 7: Plot of $p_{L}(L, t)$ (in Pa) for the low-fill example with $L=1.2 \mathrm{~m}, d=0.6 \mathrm{~m}$, $h_{1}^{(0)}=0.12 \mathrm{~m}, \omega=2.839 \mathrm{~s}^{-1}, \epsilon=6 \times 10^{-2} \mathrm{~m}, \delta=10^{-2} \mathrm{~m}^{2} \mathrm{~s}^{-1}, \Delta t=10^{-4} \mathrm{~s}$ and $M=400$. The dashed line gives the experimental results of [44] in which we have stretched their time variable in order to equate the forcing frequencies.

simulation, but the shallow-water approximation does a good job at capturing both the qualitative and quantitative pressure variation. The only minor difference is the shallowwater result has a more gradual drop in pressure from its maximum value to its minimum value, thus there is a more gradual fluid motion in the simulation, compared to the more rapid fall of the fluid at $x=L$ in the experiment.

\subsection{High-fill example}

Here we consider a rectangular vessel with length $L=0.8 \mathrm{~m}$, height $d=0.5 \mathrm{~m}$, filled to a quiescent height $h_{1}^{(0)}=0.4 \mathrm{~m}\left(h_{2}^{(0)}=0.1 \mathrm{~m}\right)$ which has $F(t)=\epsilon \cos (\omega t)$, and is forced to oscillate with frequency $\omega=7.760 \mathrm{~s}^{-1}$ from (4.23) with an amplitude $\epsilon=10^{-2} \mathrm{~m}$.

In figure 8 and table 3 , we consider the convergence of the semi-Lagrangian scheme for this high-fill example with smoothing parameter $\delta=10^{-3} \mathrm{~m}^{2} \mathrm{~s}^{-1}$ and $H^{*}=10^{-15} \mathrm{~m}$. In particular in figures 8 (a)-8(c) are plots of $h_{1}(0, t), h_{1}(x, 5)$ and $h_{1}(x, 12)$ respectively for $\Delta t=10^{-4} \mathrm{~s}$ and $M=200,300,400,500$ labelled 1-4 respectively. In panel (a) the $M=200$ result has a lower interface height at $x=0$ in the troughs of the solution, while the other three results are almost indistinguishable from each other suggesting the scheme is converging in the large $M$ limit. This can also be seen in figures 8 (b) and 8 (c) where for $M=400$ and $M=500$ the two interface results are very similar, while for $M=200$ and $M=300$ the interfaces are a similar shape, but are slightly time delayed with the speed of the hydraulic jump not agreeing. In figure $8(\mathrm{~d})$ we consider $I(\Delta t, M, 0)$ and we see a 


\begin{tabular}{|l|r|l|l||l|}
\hline$M$ & $\Delta t$ & $I(\Delta t, M, 0)$ & $h_{1}(L, 12)$ & $x_{w}(t=5)$ \\
\hline 100 & $2 \times 10^{-4}$ & 5.97358 & 0.30462 & 0.600 \\
100 & $10^{-4}$ & 5.97440 & 0.30462 & 0.592 \\
100 & $5 \times 10^{-5}$ & 5.98420 & 0.30386 & 0.616 \\
100 & $2 \times 10^{-5}$ & 5.99197 & 0.32264 & 0.744 \\
100 & $10^{-5}$ & 5.99487 & 0.30772 & 0.664 \\
200 & $2 \times 10^{-4}$ & 5.97473 & 0.37186 & 0.616 \\
200 & $10^{-4}$ & 5.97387 & 0.30112 & 0.616 \\
200 & $5 \times 10^{-5}$ & 5.97416 & 0.30167 & 0.620 \\
200 & $2 \times 10^{-5}$ & 5.97441 & 0.30192 & 0.596 \\
200 & $10^{-5}$ & 5.97434 & 0.30207 & 0.612 \\
300 & $10^{-4}$ & 5.97767 & 0.29458 & 0.616 \\
300 & $5 \times 10^{-5}$ & 5.97544 & 0.29893 & 0.616 \\
300 & $2 \times 10^{-5}$ & 5.97530 & 0.29936 & 0.611 \\
300 & $10^{-5}$ & 5.97534 & 0.29951 & 0.597 \\
400 & $10^{-4}$ & 5.97929 & 0.29317 & 0.620 \\
400 & $5 \times 10^{-5}$ & 5.97867 & 0.29375 & 0.620 \\
400 & $2 \times 10^{-5}$ & 5.97799 & 0.29438 & 0.602 \\
400 & $10^{-5}$ & 5.97706 & 0.29595 & 0.610 \\
500 & $5 \times 10^{-5}$ & 5.97970 & 0.29306 & 0.619 \\
500 & $2 \times 10^{-5}$ & 5.97916 & 0.29346 & 0.608 \\
500 & $10^{-5}$ & 5.97881 & 0.29362 & 0.606 \\
\hline
\end{tabular}

Table 3: Table of convergence data to accompany figure 8 ,

similar behaviour as for the low-fill example (which is supported by the values in table 3), with the scheme converging slowly for large $M$ and small $\Delta t$. In table 3 we also consider the value $x_{w}$, which is the 'water line' value, at $t=5 \mathrm{~s}$. This value corresponds to the $x$ value at which the free-surface intersects with the rigid lid, i.e. when $h_{2}=H^{*}$. For the free-surface profiles in figure 8(b) it is worth highlighting that when the free-surface wets the rigid lid, the shallow-water model predicts just one waterline point, while in experiments [35] an air bubble is pinched off in the top corner of the vessel. Theoretically and numerically predicting the existence of this air bubble requires using a compressible potential flow theory in which vertical flow velocities and accelerations are significant. The existence of this bubble causes small oscillations to occur in the pressure field which will not occur in our model [46, 35, 47]. For the remainder of the results in this section we set $M=400$ and $\Delta t=10^{-4} \mathrm{~s}$ in order to compromise between computational speed and accuracy.

The dependency of the scheme on the model parameters $\delta$ and $H^{*}$ are considered in figure 9 with the corresponding data given in table 4 . The results in panels (a) and (b) show that, again with $\delta=10^{-3}$ or $10^{-2} \mathrm{~m}^{2} \mathrm{~s}^{-1}$ the results are very similar, but $\delta=10^{-1} \mathrm{~m}^{2} \mathrm{~s}^{-1}$ is again too large and too much energy is removed from the fluid motion. Varying the model parameter $H^{*} \in\left[10^{-5}, 10^{-15}\right]$ has only a small effect on the results, as seen in figure 9(c) where we plot a close up of the interface at $t=7 \mathrm{~s}$. This corresponds to a time when the interface is in contact with the rigid lid. Here we see that for $H^{*}=$ $10^{-15}, 10^{-10}, 10^{-8}$ and $10^{-5} \mathrm{~m}$, the interface difference is small and is confined to a region close to the lid. Based upon these results we choose to use $\delta=10^{-3} \mathrm{~m}^{2} \mathrm{~s}^{-1}$ and $H^{*}=$ 

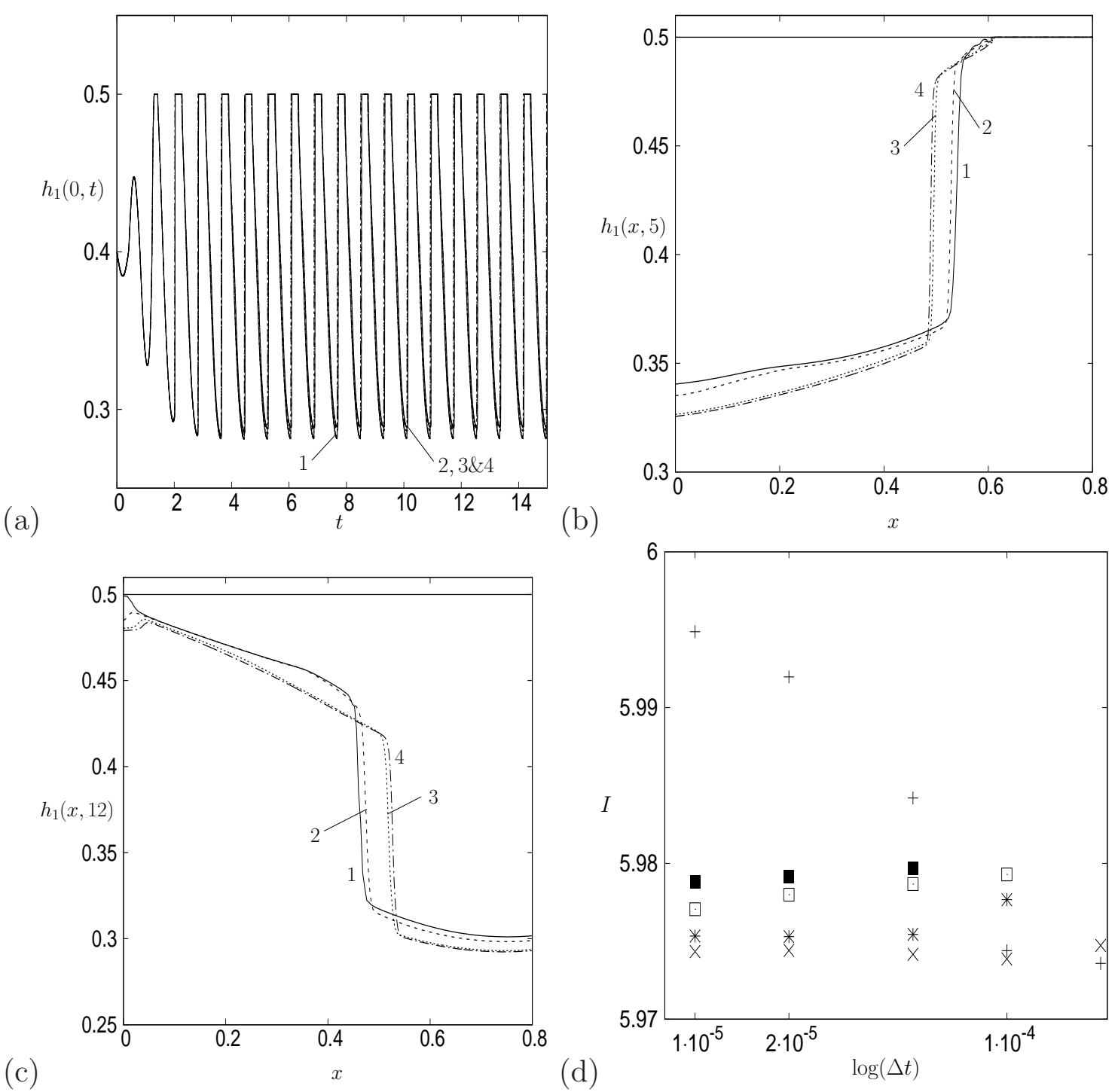

Figure 8: Plot of the convergence properties of the high-fill example with $L=0.8 \mathrm{~m}, d=$ $0.5 \mathrm{~m}, h_{1}^{(0)}=0.4 \mathrm{~m}, \omega=7.760 \mathrm{~s}^{-1}, \epsilon=10^{-2} \mathrm{~m}$ and $\delta=10^{-3} \mathrm{~m}^{2} \mathrm{~s}^{-1}$. The Panels plot (a) $h_{1}(0, t)$, (b) $h_{1}(x, 5)$ and (c) $h_{1}(x, 12)$ all with $\Delta t=2 \times 10^{-4}$ and $M=200,300,400,500$ numbered 1-4 respectively, while panel (d) plots $I(\Delta t, M, 0)$ with $(+) M=100,(\times)$ $M=200,(*) M=300,(\square) M=400$ and (口) $M=500$.

\begin{tabular}{|l|r|l|l||l|}
\hline$\delta$ & $H^{*}$ & $I(\Delta t, M, 0)$ & $h_{1}(0,13)$ & $x_{w}(t=7)$ \\
\hline $10^{-1}$ & $10^{-15}$ & 6.02117 & 0.37465 & - \\
$10^{-2}$ & $10^{-15}$ & 5.99029 & 0.36800 & 0.208 \\
$10^{-3}$ & $10^{-15}$ & 5.97930 & 0.37086 & 0.212 \\
$10^{-3}$ & $10^{-10}$ & 5.97920 & 0.37088 & 0.218 \\
$10^{-3}$ & $10^{-8}$ & 5.97918 & 0.37087 & 0.214 \\
$10^{-3}$ & $10^{-5}$ & 5.97911 & 0.37090 & 0.220 \\
\hline
\end{tabular}

Table 4: Table of convergence data to accompany figure 9 , 


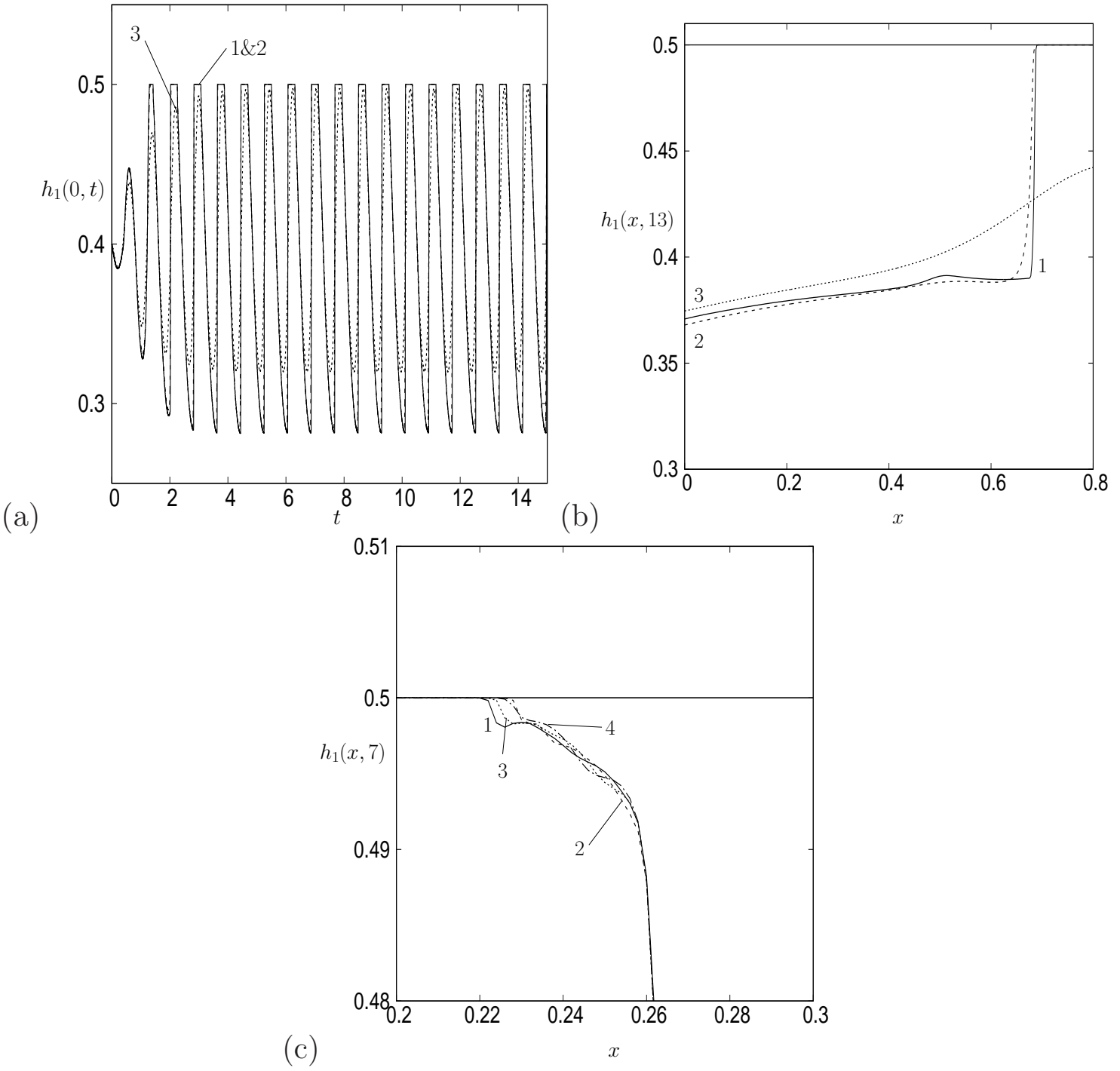

Figure 9: Plot of (a) $h_{1}(0, t)$ and (b) $h_{1}(x, 13)$ for the high-fill example when $L=$ $0.8 \mathrm{~m}, d=0.5 \mathrm{~m}, h_{1}^{(0)}=0.4 \mathrm{~m}, \omega=7.760 \mathrm{~s}^{-1}, \epsilon=10^{-2} \mathrm{~m}$ with $H^{*}=10^{-15} \mathrm{~m}$ and $\delta=10^{-3}, 10^{-2}, 10^{-1} \mathrm{~m}^{2} \mathrm{~s}^{-1}$ numbered 1-3 respectively. In panel (c) we plot $h_{1}(x, 7)$ for $\delta=10^{-3}$ and $H^{*}=10^{-15}, 10^{-10}, 10^{-8}$ and $10^{-5}$ m numbered $1-4$ respectively.

$10^{-15} \mathrm{~m}$ for the remaining results in this section.

One concern with the semi-Lagrangian scheme is how well it copes with a flow that periodically changes direction, as many problems for which it is used often have a preferred direction. In figure 10 comparing two simulations, one with $\epsilon=10^{-2} \mathrm{~m}$ and another with $-\epsilon=10^{-2} \mathrm{~m}$ (i.e. initially moving in the opposite direction), we observe that the forms of $h_{1}(0, t)$ and $h_{1}(x, 11)$ are identical (Note, we have plotted $h_{1}(L, t)$ and $h_{1}(L-x, 11)$ for the $-\epsilon=10^{-2} \mathrm{~m}$ simulation for comparison). In fact for all time values for this simulation the interface profiles are identical, thus this semi-Lagrangian approach is suitable for simulating this type of sloshing problem.

Finally, for this high-fill wetting/drying example we make a comparison between our simulation and experimental data of [45]. Note that their forcing magnitude was $2 \times$ $10^{-2} \mathrm{~m}$ and their frequency was $\omega=5.938 \mathrm{~s}^{-1}$ (a non-shallow-water case), compared to 

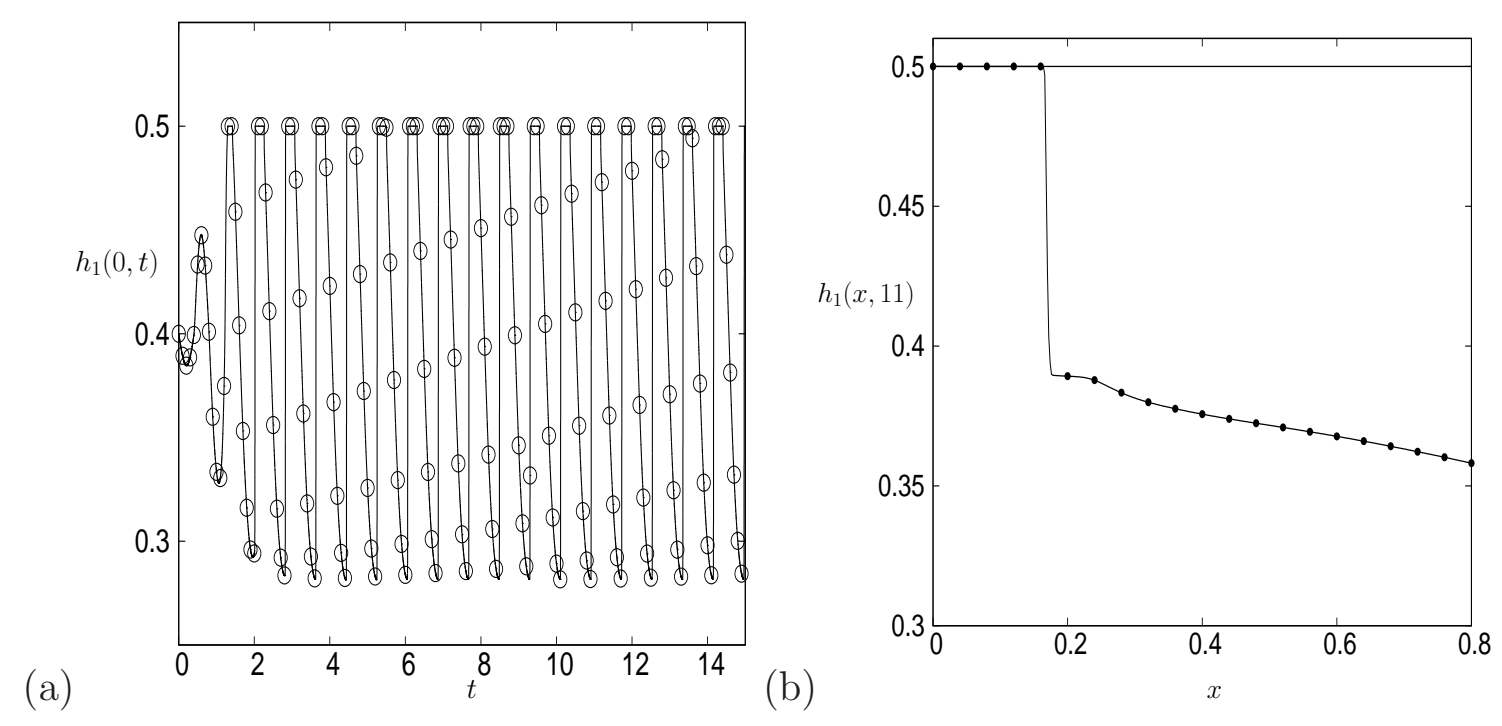

Figure 10: Plot of (a) $h_{1}(0, t)$ and (b) $h_{1}(x, 11)$ for the high-fill example when $L=0.8 \mathrm{~m}$, $d=0.5 \mathrm{~m}, h_{1}^{(0)}=0.4 \mathrm{~m}, \omega=7.760 \mathrm{~s}^{-1}$, with $\epsilon=10^{-2} \mathrm{~m}$ and $-\epsilon=10^{-2} \mathrm{~m}$. For the $-\epsilon=10^{-2} \mathrm{~m}$ results we plot $h_{1}(L, t)$ (circles) and $h_{1}(L-x, 11)$ (dots) in panels (a) and (b) respectively.

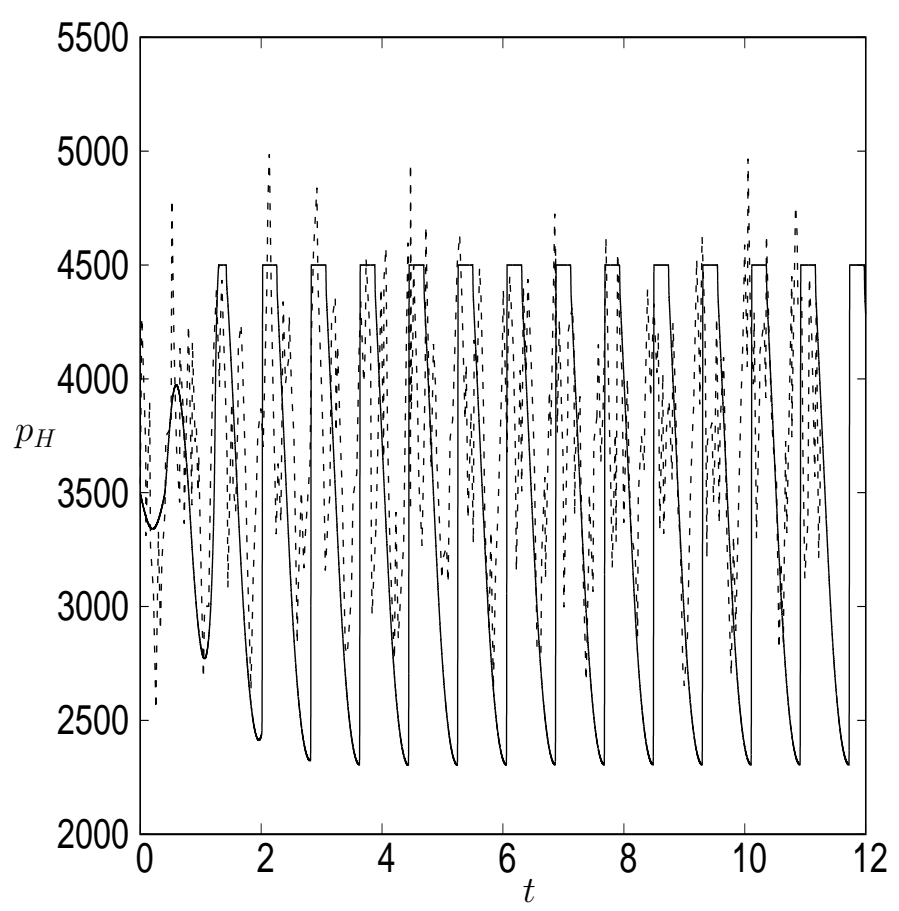

Figure 11: Plot of $p_{H}(0, t)$ (in Pa) for the high-fill example with $L=0.8 \mathrm{~m}, d=0.5 \mathrm{~m}$, $h_{1}^{(0)}=0.4 \mathrm{~m}, \omega=7.760 \mathrm{~s}^{-1}$ and $\epsilon=10^{-1} \mathrm{~m}$. The dashed line gives the experimental results of [45] in which we have stretched their time variable in order to equate the forcing frequencies.

$\epsilon=10^{-1} \mathrm{~m}$ and $\omega=7.760 \mathrm{~s}^{-1}$ for our simulation. Therefore we again stretch the time values for the experimental data to equate the forcing frequencies in order to make a 
comparison. Note that as in $\$ 4.1$ we do this because we need to force our vessel at a resonant frequency in order to generate a large free-surface response. Also, we are not comparing equivalent forcing amplitudes, but it is known that shallow-water simulations overestimate the wave amplitudes in non-shallow-water conditions, when compared to non-shallow-water simulations and data, hence our smaller amplitude should still give a good qualitative comparison with the data, which is our aim. The experimental data is taken from a pressure sensor placed at $x=0,0.0525 \mathrm{~m}$ from the bottom of the vessel, hence the pressure at the sensor for our result is

$$
p_{H}(0, t)=\rho_{1} g\left(h_{1}(0, t)-0.0525\right)+\rho_{2} g h_{2}(0, t) .
$$

The comparison in figure 11 shows reasonable agreement between simulation and experiment. The experimental time series is taken mid-cycle, hence the poor agreement with the simulation initially, but after this initial time period $t \in[0,1]$, the peaks in the pressure agree relatively well with the points where $p_{H}(0, t)$ reaches, and leaves, the value $4500 \mathrm{~Pa}$, which corresponds to times when the upper rigid lid is initially wetted, and then dries completely, on a forcing cycle. As our simulation is a shallow-water simulation and the experiment is not in this limit, we also see that we over-predict the troughs in the pressure for this high-fill case, but as our aim for this semi-Lagrangian scheme is to investigate shallow-water sloshing in wave energy converters these results are acceptable.

\section{Conclusions and discussion}

This article contains a numerical scheme to investigate forced rectilinear sloshing of two shallow-water, inviscid, incompressible, immiscible fluids in a rectangular vessel with a horizontal rigid lid. The vessel was forced to oscillate with the lowest resonant frequency of the system such that the amplitude of the lower, more dense, fluid increases in magnitude until nonlinear effects become significant. The numerical scheme devised in this article was based upon the conservative quasi-monotone semi-Lagrangian (CQMSL) algorithm which conserves fluid mass and generates no spurious oscillations into the solution. The main advantage of using the CQMSL-form of the semi-Lagrangian method, over other shallow-water schemes in the literature is that wetting and drying of the upper rigid lid (or the bottom of the vessel) can be captured.

We considered two examples based upon water/air $\left(\rho_{2} / \rho_{1} \approx 10^{-3}\right)$ experimental setups: a low-fill example where the upper rigid lid remains dry, and a high-fill example where the upper lid undergoes wetting/drying over each period of the forcing. The results of the low-fill example were compared to the Lagrangian Particle Path (LPP) code of [3] and the experiments of [44]. The low and moderate amplitude simulations were found to be in excellent agreement with the LPP simulations: while the larger amplitude simulations were in good agreement with the experiments. The peak and trough values of the pressure variation quantitatively agree with experimental measurements, although the semi-Lagrangian scheme does not have the high-frequency oscillations in pressure present in the experiment (see figure 77). In the high-fill example the fluid interface runs up the side-walls of the vessel and subsequently wets the upper rigid lid. When the vessel is then forced to move in the opposite direction the rigid lid dries and the bulk of the fluid sloshes across the vessel and up the opposite side wall. This periodic wetting/drying of the rigid lid could not be captured by the LPP scheme used to validate the semi-Lagrangian scheme 
in the low-fill example. Results for this example were in reasonable qualitative agreement with the experimental results of [45].

The motivation for this idealised problem is to use the proposed scheme on the waveenergy converter (WEC) based upon the patent of Offshore Wave Energy Ltd (OWEL), in which the power-take-off mechanism relies on pushing trapped air, via a wetted upper surface, through the power-take-off unit. As noted in the introduction, experimental and CFD studies of the OWEL WEC show the interior 3D motion can be modelled as predominantly 2D, with some inner topography which is used to focus the waves. The topography can be included in the present numerical formulation by using the twolayer equivalent of the shallow-water equations analysed in [26]. Modelling the WEC also means incorporating influx-efflux boundary conditions at the side-walls, which would model waves entering the converter at one end and exiting via the extraction route at the other end. The mechanism for introducing this is to modify (2.10) such that the combined flux is some given time-dependent function, as successfully implemented in [25]. Extending the current semi-Lagrangian scheme to include rotation (pitch, and potentially roll and yaw) and vertical translations together with the horizontal translations considered here is also of great interest.

\section{Acknowledgements}

The authors would like to thank Y. G. Chen for supplying the experimental data used in this publication [48].

\section{- Appendix -}

\section{A Derivation of $(2.8)$}

In this appendix we highlight the derivation of (2.8) and the consequences of this derivation in (2.9) . Firstly, note that (2.11) is the momentum equation derived by combining (2.4) and (2.6) and eliminating $h_{1}$ and $u_{1}$. Thus at $x=0$ and $L, u_{2}=0$ for all time, hence $u_{2 t}=0$. Substituting these into (2.11) leaves

$$
h_{2}\left(d-h_{2}\right)\left(g h_{2 x}-\ddot{F}\right)=0 .
$$

Clearly $h_{2}=0$ and $d-h_{2}=h_{1}=0$ are solutions. For the other solution, we require the interface to have a $90^{\circ}$ contact angle with the side wall at these points because our problem is inviscid and does not include surface tension effects, thus $h_{2 x}=0$. Then for consistency we get condition (2.9). Differentiating (1.1) with respect to $x$ then gives that $h_{1 x}=0$. Finally note that substituting this information into (2.6) gives $p_{x}=0$ at $x=0, L$. 


\section{References}

[1] M. Leybourne, W. Batten, A. S. Bahaj, J. O’Nians and N. Minns, Experimental and Computational Modelling of the OWEL Wave Energy Converter, 3rd International Conference on Ocean Energy, Bilbao, Spain, (2010).

[2] A. Balzano, Evaluation of methods for numerical simulation of wetting and drying in shallow water flow models, Coastal Engineering, 34 (1998) 83-107.

[3] M. R. Turner, T. J. Bridges and H. Alemi Ardakani, Lagrangian particle path formulation of multilayer shallow-water flows dynamically coupled to vessel motion, J. Eng. Maths, 106(1) (2017) 75-106.

[4] J. Frank and S. Reich, A particle-mesh method for the shallow water equations near geostrophic balance, J. Comp. Phys., 180(2) (2002) 407-426.

[5] C. J. Cotter, J. Frank, S. Reich, Hamiltonian Particle-Mesh method for two-layer shallow-water equations subject to the rigid-lid approximation, SIAM J. Appl. Dyn. Syst., 3(1) (2004) 69-83.

[6] M. H. Mawson and Great Britain. Meteorological Office, Implementation of semiLagrangian advection in the next generation U.K. Met Office unified model Bracknell, Berkshire : Meteorological Office Atmospheric circulation - Mathematical models; Numerical weather forecasting (1995).

[7] T. Davies, M. J. P. Cullen, A. J. Malcolm, M. H. Mawson, A. Staniforth, A. A. White and N. Wood, A new dynamical core for the Met Office's global and regional modelling of the atmosphere, Q. J. Royal Meteorol. Soc., 131(608) (2005) 1759-1782.

[8] M. Diamantakis, The semi-Lagrangian technique in atmospheric modelling: current status and future challenges, In ECMWF Seminar in numerical methods for atmosphere and ocean modelling, (2013) 183-200.

[9] A. Staniforth and J. Côté, Semi-Lagrangian integration schemes for atmospheric models - A review, Mon. Weather Rev., 119(9) (1991) 2206-2223.

[10] C. J. Smith, The Semi-Lagrangian Method in Atmospheric Modelling, Ph.D. Thesis, University of Reading, (2000).

[11] C. Hu and M. Sueyoshi, Numerical simulation and experiment on dam break problem, J. Mar. Sci. Appl., 9(2), (2010) 109-114.

[12] R. Tanaka, T. Nakamura T. Yabe, Constructing exactly conservative scheme in a non-conservative form, Comput. Phys. Commun., 126(3) (2000) 232-243.

[13] F. Xiao and T. Yabe, Completely conservative and oscillationless semi-Lagrangian schemes for advection transportation, J. Comp. Phys., 170(2) (2001) 498-522.

[14] T. Yabe, R. Tanaka, T. Nakamura and F. Xiao, An exactly conservative semiLagrangian scheme (CIP-CSL) in one dimension, Mon. Weather Rev., 129(2) (2001) 332-344. 
[15] T. Nakamura, R. Tanaka, T. Yabe and K. Takizawa, Exactly conservative semiLagrangian scheme for multi-dimensional hyperbolic equations with directional splitting technique, J. Comp. Phys., 174(1) (2001) 171-207.

[16] K. Takizawa, T. Yabe and T. Nakamura, Multi-dimensional semi-Lagrangian scheme that guarantees exact conservation, Comput. Phys. Commun., 148(2) (2002) 137159.

[17] M. Lentine, J. T. Grétarsson and R. Fedkiw, An unconditionally stable fully conservative semi-Lagrangian method, J. Comp. Phys., 230(8) (2011) 2857-2879.

[18] R. Bermejo and J. Conde, A conservative quasi-monotone semi-Lagrangian scheme, Mon. Weather Rev., 130(2) (2002) 423-430.

[19] L. V. Ovsyannikov, Two-layer shallow water model, Journal of Applied Mechanics and Technical Physics, 20(2) (1979) 127-135.

[20] G. A. Lawrence, On the hydraulics of Boussinesq and non-Boussinesq two-layer flows, J. Fluid Mech., 215 (1990) 457-480.

[21] F. Menzaque, P. Milewski, R. Rosales, E. Tabak and C. Turner, Nonlinear stability of two-layer flows, Comm. Math. Sci., 2(3) (2004) 427-442.

[22] T. J. Bridges and N. M. Donaldson, Reappraisal of criticality for two-layer flows and its role in the generation of internal solitary waves, Phys. Fluids, 19(7) (2007) 072111.

[23] J. Kim and R. J. LeVeque, Two-layer shallow water system and its applications, In Proceedings of the Twelfth International Conference on Hyperbolic Problems, Maryland, (2008).

[24] J. Esler and J. D. Pearce, Dispersive dam-break and lock-exchange flows in a twolayer fluid, J. Fluid Mech., 667 (2011) 555-585.

[25] H. Alemi Ardakani, T. J. Bridges and M. R. Turner, Adaptation of f-wave finite volume methods to the two-layer shallow-water equations in a moving vessel with a rigid-lid, J. Comput. Appl. Math. 296 (2016) 462-479.

[26] H. Alemi Ardakani, T. J. Bridges and M. R. Turner, Shallow-water sloshing in a moving vessel with variable cross-section and wetting-drying using an extension of George's well-balanced finite volume solver, J. Comput. Phys., 314 (2016) 590-617.

[27] D. L. George, Finite volume methods and adaptive refinement for tsunami propagation and inundation, Ph.D. Thesis, University of Washington, (2006).

[28] P. G. Baines, Topographic Effects in Stratified Flows, Cambridge University Press (1998).

[29] O. Bokhove, Flooding and Drying in Discontinuous Galerkin Finite-Element Discretizations of Shallow-Water Equations. Part 1: One Dimension, J. Sci. Comp., 22 (2005) 47-82. 
[30] S. C. Medeiros and S. C. Hagen, Review of wetting and drying algorithms for numerical tidal flow models, Int. J. Numer. Meth. Fluids, 71(4) (2013) 473-487.

[31] N. E, Mikelis, J. K. Miller and K. V. Taylor, Sloshing in partially filled liquid tanks and its effect on ship motions: numerical simulations and experimental verification, Trans. Roy. Inst. Naval Architects, 126 (1984).

[32] P. C. Sames, D. Marcouly and T. E. Schellin, Sloshing in rectangular and cylindrical tanks, J. Ship Res., 46(3) (2002) 186-200.

[33] A. Souto-Iglesias, L. Delorme, L. Pérez-Rojas and S. Abril-Pérez, Liquid moment amplitude assessment in sloshing type problems with smooth particle hydrodynamics, Ocean Eng., 33(11-12) (2006) 1462-1484.

[34] Y. G. Chen, K. Djidjeli and W. G. Price, Numerical simulation of liquid sloshing phenomena in partially filled containers, Comput. Fluids, 38(4) (2009) 830-842.

[35] Y. G. Chen and W. G. Price, Numerical simulation of liquid sloshing in a partially filled container with inclusion of compressibility effects, Phys. Fluids, 21(11) (2009) 112105.

[36] H. Alemi Ardakani, T. J. Bridges and M. R. Turner, Dynamic coupling between horizontal vessel motion and two-layer shallow-water sloshing, J. Fluid Struct. 59 (2015) 432-460.

[37] M. Antuono, B. Bouscasse, A. Colagrossi and C. Lugni, Two-dimensional modal method for shallow-water sloshing in rectangular basins, J. Fluid Mech., 700 (2012) 419-440.

[38] F. N. Fritsch and R. E. Carlson, Monotone piecewise cubic interpolation, SIAM J. Numer. Anal., 17(2) (1980) 238-246.

[39] M. Antuono, A. Colagrossi, S. Marrone and D. Molteni, Free-surface flows solved by means of SPH schemes with numerical diffusive terms, Comput. Phys. Comm. 181(3) (2010) 532-549.

[40] M. Antuono, A. Colagrossi, S. Marrone and C. Lugni, Propagation of gravity waves through an SPH scheme with numerical diffusive terms, Comput. Phys. Comm. 182(4) (2011) 866-877.

[41] M. Antuono, A. Bardazzi, C. Lugni and M. Brocchini, A shallow-water sloshing model for wave breaking in rectangular tanks, J. Fluid Mech., 746 (2014) 437-465.

[42] H. Alemi Ardakani and M. R. Turner, Numerical simulations of dynamic coupling between shallow-water sloshing and horizontal vessel motion with baffles, Fluid Dyn. Res., 48(3) (2016) 035504.

[43] R. Bermejo and A. Staniforth, The conversion of semi-Lagrangian advection schemes to quasi-monotone schemes, Mon. Weather Rev., 120(11) (1992) 2622-2632. 
[44] M. Hinatsu, Y. Tsukada, R. Fukasawa and Y. Tanaka, Two-phase flows for joint research, In Proceedings of the SRI-TUHH Mini Workshop on Numerical Simulation of Two-Phase Flows, edited by M. Hinatsu (National Maritime Research Institute Japan, Tokyo), (2001).

[45] D. H. Kang and Y. B. Lee, Summary report of sloshing model test for rectangular model, Daewoo Shipbuilding \& Marine Engineering Co., Ltd. South Korea, 001 (2005).

[46] S. Zhang, D. K. P. Yue and K. Tanizawa, Simulation of plunging wave impact on a vertical wall, J. Fluid Mech. 327 (1996) 221-254

[47] B. C. Abrahamsen and O. M. Faltinsen, A numerical model of an air pocket impact during sloshing, Appl. Ocean Res., 37 (2012) 54-71.

[48] Y. G. Chen, Private communication, (2018). 